\title{
Pruriception and neuronal coding in nociceptor subtypes in human and nonhuman
}

\section{primates}

Amanda H Klein ${ }^{1,4}$, Hans Jürgen Solinski ${ }^{2,5}$, Nathalie M Malewicz ${ }^{3,6}$, Hada Fong-ha Ieong ${ }^{3}$, Steven G Shimada ${ }^{3}$, Timothy V Hartke ${ }^{1}$, Matthew Wooten ${ }^{1}$, Gang Wu${ }^{1}$, Mark A. Hoon ${ }^{2}$, Robert H LaMotte ${ }^{3}$, Matthias Ringkamp ${ }^{1}$

${ }^{1}$ Department of Neurosurgery, Neurosurgery Pain Research Institute, School of Medicine, Johns Hopkins University, 600 N Wolfe St, Baltimore, MD 21287

${ }^{2}$ Molecular Genetics Section, National Institute of Dental and Craniofacial Research/NIH, 35 Convent Drive, Bethesda, MD 20892, USA.

${ }^{3}$ Department of Anesthesiology, School of Medicine, Yale University, 333 Cedar St., New Haven, CT 06520, USA

${ }^{4}$ Department of Pharmacy Practice and Pharmaceutical Sciences, University of Minnesota, Duluth, MN 55812, USA

${ }^{5}$ Department of Experimental Pain Research, Medical Faculty Mannheim, University of Heidelberg, Mannheim, Germany

${ }^{6}$ Department of Anesthesiology, Intensive Care Medicine and Pain Management, Medical Faculty of Ruhr-University Bochum, BG University Hospital Bergmannsheil, Bochum, Germany

Correspondence should be sent to: RHL (robert.lamotte@yale.edu), MR (platelet@jhmi.edu)

\section{Acknowledgements}

This study was supported by NIH grants R01 AR070875 (M.R., R.H.L.) and K01 DA042902 (A.H.K); by the German Research Foundation (DFG): project grant FOR 2690 (H.J.S.) and Research Fellowship grant 326726541 (NMM), the intramural research program of the National Institute of Dental and Craniofacial Research, National Institutes of Health, project ZIADE000721-18 (MAH), and the Neurosurgery Pain Research Institute at the Johns Hopkins University. Human tissue was obtained from the NIH NeuroBioBank at the University of Maryland, Baltimore, MD.

Conflicts of interests: The authors declare no competing financial interests. 


\section{$\underline{\text { Abstract }}$}

In human, intradermal administration of $\beta$-alanine (ALA) and bovine adrenal medulla peptide 822 (BAM8-22) evokes the sensation of itch. Currently, it is unknown which human dorsal root ganglion (DRG) neurons express the receptors of these pruritogens, MRGPRD and MRGPRX1 respectively, and which cutaneous afferents these pruritogens activate in primate. In situ hybridization studies revealed that MRGPRD and MRGPRX1 are co-expressed in a subpopulation of TRPV1+ human DRG neurons. In electrophysiological recordings in nonhuman primates (Macaca nemestrina), subtypes of polymodal C-fiber nociceptors are preferentially activated by ALA and BAM8-22, with significant overlap. When pruritogens ALA, BAM8-22 and histamine, that activate different subclasses of C-fiber afferents, are administered in combination, human volunteers report itch and nociceptive sensations similar to those induced by a single pruritogen. Our results provide evidence for differences in pruriceptive processing between primates and rodents, and do not support the spatial contrast theory of coding of itch and pain. 


\section{Introduction}

The sensations of itch and pain serve a similar purpose, which is to alert the organism of potentially harmful external threats. Although the sensations and behavioral responses of itch and pain are quite distinct (such as scratching vs withdrawal, rubbing or guarding), they are closely linked as they appear to be mediated by either identical or functionally similar primary afferents.

In mice, pruritogens activate at least three populations of neurons (Liu et al., 2009; Liu et al., 2012; Solinski et al., 2019b). Among these, two neuronal populations express Mas-related Gprotein-coupled receptors (Mrgpr), namely Mrgprc and Mrgprd, in a non-overlapping fashion (Zylka et al., 2003), and intradermal administration of their respective agonist, bovine adrenal medulla peptide 8-22 (BAM) and $\beta$-alanine (ALA), results in scratching behavior. The third population expresses natriuretic polypeptide b $(N p p b)$ and mediates mast cell-induced itch. Neurons expressing Mrgprc or Nppb also express the histamine receptor Hrh1 and likely mediate scratching behavior induced by histamine (HIS).

In humans, HIS and the nonhistaminergic pruritogens ALA and BAM8-22 each elicit itch and nociceptive sensations (Sikand et al., 2009; Sikand et al., 2011b; Liu et al., 2012; Sikand et al., 2011a). HIS preferentially activates mechanically insensitive C-fiber afferents (C-MIAs) in human (Schmelz et al., 1997) and nonhuman primates (Wooten et al., 2014). In these, ALA preferentially excites QCs, a subtype of mechanoheat-sensitive, polymodal C-fiber (CMH) nociceptor that adapts quickly to noxious heat as opposed to those that adapt slowly (SCs), and C-MIAs are unresponsive to ALA(Wooten et al., 2014). The type of afferent nerve fiber activated by BAM8-22, an agonist of MRGPRX1 in primate (Lembo et al., 2002), is currently unclear. Based on the non-overlapping expression of Mrgprd and Mrgprc in mouse DRG 
neurons and the preferential activation of QC-fibers by ALA in nonhuman primate, one might expect, however, that in primate MRGPRD and MRGPRX1 are similarly expressed in non overlapping DRG neurons and that ALA and BAM8-22 activate different neuronal afferent populations.

In primate, including humans, multiple types of nociceptive primary afferents including unmyelinated and myelinated nerve fibers appear to have a role in itch sensation (Schmelz et al., 1997; Johanek et al., 2007; Johanek et al., 2008; Namer et al., 2008; Ringkamp et al., 2011; Wooten et al., 2014). Moreover, HIS and nonhistaminergic pruritogens activate distinct sets of 1) nociceptive primary afferents, including mechano-insensitive C fibers (C-MIAs), CMHs, mechanosensitive A-fiber nociceptors (A-MSAs) and 2) spinothalamic projection neurons (Schmelz et al., 1997; Johanek et al., 2008; Namer et al., 2008; Wooten et al., 2014; Davidson et al., 2007). The signaling of pruritic stimuli by nociceptive afferents that are also thought to encode nociceptive mechanical, thermal and chemical stimuli (Ringkamp et al., 2013) poses a conundrum regarding the neuronal coding of these distinct sensations in the nervous system. Among the models that have been proposed to explain this conundrum (for review see (LaMotte et al., 2014; Carstens et al., 2020)), the spatial contrast model posits that neuronal activity from a spatially restricted set of nociceptive afferents is interpreted as 'itch', whereas activity from a spatially larger pool of afferents is interpreted as ‘pain’ (Namer et al., 2008; Namer \& Reeh, 2013; Steinhoff et al., 2019). However, whether co-activation of different sets of primary afferents by pruritogens that excite different classes of afferents leads to reduced itch and/or increased nociceptive sensation has not been tested in human.

Here we probed the relationship between the expression of receptors for ALA and BAM8-22 in postmortem human DRGs and the physiological responses of these pruritogens in 
single cutaneous nerve fibers of nonhuman primate. We found that human DRG neurons coexpress both, MRGPRD and MRGPRX1, and also transient receptor potential channels from the vanilloid subfamily V member 1 (TRPV1). Further, injection of ALA and BAM8-22 into the receptive fields of nociceptors in nonhuman primate revealed that QCs respond to ALA, and SCs preferentially respond to BAM8-22, with a large degree of overlap. In accompanying psychophysical studies, we studied the effects of individual pruritogens and their combinations and recorded the reported itch, nociceptive sensations and dysesthesias in human subjects. Since we know at least three pruritogens that can preferentially activate QCs, SCs, and C-MIAs, we additionally tested the hypothesis that the co-activation of the different sets of primary afferents by ALA, BAM8-22, and HIS, would enhance itch and/or nociceptive sensations and dysesthesias in humans. In addition, in primates, HIS and cowhage activate largely non-overlapping spinothalamic tract (STT) neurons (Davidson et al., 2012). Therefore, by combining ALA, BAM8-22, and HIS, we tested psychophysically whether activating both types of STT neurons would result in altered itch or nociceptive sensations.

\section{$\underline{\text { Results }}$}

\section{MRGPRX1 and MRGPRD have overlapping expression patterns in human DRG}

Whether MRGPRX1 and MRGPRD are expressed in non-overlapping DRG neurons in primate, similar to what has been previously reported in mouse for Mrgprc and Mrgprd (Zylka et al., 2003), is currently unclear. We therefore investigated the expression of MRGPRX1 and MRGPRD in human DRG using double-label in situ hybridization (ISH). We found that MRGPRX1 and MRGPRD were co-expressed in the same neurons (Figure 1A). Specifically, on average $89.6 \pm 1.5 \%$ of DRG neurons positive for MRGPRD in a given donor were also positive 
for MRGPRX1 (380/426 neurons from 4 donors combined), and $93.9 \pm 2.0 \%(380 / 407)$ of DRG neurons positive for MRGPRX1 were also positive for MRGPRD. Previously it has been reported in a Mrgprd reporter mouse line, that Mrgprd+ neurons were TRPV1 negative and nonpeptidergic CMHs (Rau et al., 2009). If findings in human DRG were comparable to mouse, then one would expect to observe no overlap between TRPV1 and MRPGRD expression. However, we found that MRGPRD neurons nearly always co-expressed TRPV1 (Figure 1B) with $95.2 \pm$ 1.6\% (479/510) of MRGPRD+ neurons being also positive for TRPV1 (Figure 1D). In the mouse, Mrgprc is expressed in a subset of capsaicin responsive neurons (Liu et al., 2009). Recent ISH data from human DRG show that all MRGPRX1+ neurons co-express NPPB, and that NPPB expressing neurons are a sub-set of TRPV1+ neurons (Solinski et al., 2019a). In agreement with these previous findings, we observed that 100\% (429/429) of MRGPRX1+ neurons were also positive for TRPV1 (Figure 1C, D). Of TRPV1+ neurons, $32.5 \pm 3.7 \%$ (429/1273) and $36.5 \pm 4.2 \%$ (479/1211) of cells also expressed MRGPRX1 and MRGPRD, respectively (Figure 1D).

\section{BAM8-22 preferentially activates SCs in nonhuman primates}

To learn more about the neurons which are activated by ALA and BAM8-22, we probed the different functional classes of neurons using teased fiber recordings from single nerve fibers in the primate (Wooten et al., 2014). Across all CMHs tested, the average number of action potentials (APs) elicited by BAM8-22 was significantly larger than the response to ALA (74.95 \pm 8.88 APs vs $47.44 \pm 6.05$ APs, respectively; paired t-test: $t_{(65)}=-2.142, p=0.0359$; Figure $2 \mathrm{~A}$ ). The response profiles for ALA and BAM8-22 across all responsive CMHs were indistinguishable, except for the first $10 \mathrm{~s}$ following the injection when BAM8-22-induced 
activity was about twice that of ALA. The duration of action potential activity was similar after BAM8-22 and ALA injection (Figure 2B).

Based on responses to a stepped heat stimulus, CMHs can be classified into two distinct subtypes, QCs and SCs (Wooten et al., 2014). Briefly, heat responses of QCs exhibit a burst of discharge at the onset of the heat stimulus with the discharge adapting during the stimulus plateau. SCs typically have a slower response onset, do not exhibit a burst at the onset of the stimulus, and the peak discharge occurs during the plateau phase of the stimulus (Supplemental Figure 1A, B). QCs and SCs can be formally separated by plotting the time of peak discharge (relative to stimulus onset) against the sum of temperature rise time plus the minimum conduction latency from skin (Supplemental Figure 1C). For 31 CMHs, the time of peak discharge fell above the line of equality (Supplemental Figure 1C, black line), indicating that the peak discharge occurred during the plateau phase of the stimulus, and these afferents were therefore classified as 'SC' (Supplemental Figure 1C, blue circles). Twenty nine CMH fibers exhibited a burst of discharge at the onset and then an adapting response, and these afferents were classified as 'QC' (Supplemental Figure 1C, red circles). For 26/29 QCs, the time of peak discharge fell below or close to the line of equality, indicating occurrence of peak discharge during or at the end of the rising phase of the temperature ramp. For the remaining three QC fibers, the time of peak discharge fell above the line of equality. Another 6 CMHs, in which the time of peak discharge fell close to the line of equality and which did not exhibit an initial burst discharge typical of a QC response, were labeled as 'unclassified’ (Supplemental Figure 1C, grey circles). The filled red and blue circles represent the data points for the specimen heat responses shown in Supplemental Figure 1A, B. 
We found that the two subpopulations for CMHs, QCs and SCs, differed in their responses to injections of ALA and BAM8-22 into their cutaneous receptive fields. The differences are shown first by the responses of a typical QC and SC fiber in Figure 3 and summarized for the two populations in Figure 4. For the specimen QC (Figure 3A), injection of ALA (top panel) and BAM8-22 (bottom panel) induced a neuronal response for about 5 minutes, and the response to ALA (81 APs) was larger than to BAM8-22 (50 APs). Vehicle (ECF, BAM8-18) did not produce any activity in this afferent beyond the injection (data not shown). In the SC-fiber, ALA administration did not result in activity outlasting the injection (Figure 3B, top panel) and the preceding ECF injection (response not shown) produced a total response of 18 APs. In contrast, injection of BAM8-22 (Figure 3B, bottom panel) produced a vigorous response of 198 APs within 5 minutes. The preceding BAM8-18 injection only produced activity during the injection (response not shown). Time courses of ALA- and BAM8-22-induced activity across the QC- and SC-populations are shown in Figure 3C and 3D, respectively. In QCs and SCs, vehicles did not evoke activity beyond the injection period. In QCs, ALA and BAM8-22 caused excitation with a similar time course (Figure 3C). For both agonists, the highest activity was observed immediately following the injection, and the activity decreased throughout the 5minute observation period. At 5 minutes post injection the number of APs in QCs over 10 seconds was $0.55 \pm 0.29$ and $1.14 \pm 0.23$ for BAM8-22 and ALA, respectively. In contrast, in SCs, only BAM8-22- induced activity outlasted the injection and was different from the response to vehicle injections (Figure 3D). The highest activity occurred during the first 10 seconds following the injection, and the response decreased within approximately 3 minutes to a level similarly seen following vehicle injection. 
To investigate further whether responses to ALA and BAM8-22 differed between QCs and SCs, the net response to BAM8-22 was plotted against that to ALA for each individual afferent (Figure 4A). For 20/29 QCs, data points fell below the diagonal line of equal responsiveness indicating that responses to ALA were larger than responses to BAM8-22. In contrast, for 26/31 SCs, responses to BAM8-22 were greater than those to ALA. In fact, ALA responses in only 7 SCs fulfilled the criterion of a positive response ( $\geq 10$ APs). One SC was unresponsive to both agonists. Responses to ALA and BAM8-22 differed significantly within and across fiber types (Figure 4B, repeated measures ANOVA (RMANOVA) with 'fiber type' as a between subjects factor and 'pruritogen' as within subject factor; interaction: $F_{(1,58)}=29.55$, $\mathrm{p}<0.001)$. Post hoc analysis revealed that for SCs $(\mathrm{n}=31)$, responses to BAM8-22 were significantly larger than those to ALA $(94.00 \pm 12.01$ APs vs $10.32 \pm 3.22$ APs, $p<0.001$, Scheffe test). In QCs ( $\mathrm{n}=29)$ responses to ALA were larger than those to BAM8-22 (83.38 \pm 8.42 APs vs $51.97 \pm 11.17$ APs, respectively), but this difference was not significant $(\mathrm{p}=0.25)$. Between populations, responses to ALA were significantly larger in QCs than SCs ( $p<0.001$, Scheffe test), whereas responses to BAM8-22 were significantly larger in SCs than QCs ( $\mathrm{p}=0.021$, Scheffe test). ALA activated 27/29 QCs and 7/31 SCs. BAM8-22 activated 21/29 QCs and 29/31 SCs. Of all QCs and SCs tested, the majority (50/60) responded to BAM8-22, and 34/60 fibers responded to ALA. 25/60 responded to both agonists, and 9/60 responded only to ALA, and 25/60 responded only to BAM8-22 (Figure 4C). Of the 6 unclassified afferents, 3 units responded to both agonists, 2 responded to ALA only, and the remaining only to BAM8-22.

Thus, BAM8-22 activated 54/66 CMHs, whereas ALA activated 39/66 fibers. Across all fibers tested, the incidence of responsiveness to BAM8-22 and ALA was significantly different $\left(\mathrm{X}_{(1)}^{2}=\right.$ 8.19, $\mathrm{p}<0.01$ ). Taken together, these findings suggest that, at the doses tested, QCs and SCs are 
preferentially activated by ALA and BAM8-22, respectively. The data imply that QCs may represent fibers expressing both MRGPRs at the peripheral terminals, but preferentially MRGPRD, whereas SCs may preferentially express MRGPRX1. To summarize, in contrast to our ISH data which suggested nearly 100\% overlap of MRGPRX1 and MRGPRD expression, we found that only about half of the CMH neurons tested responded to both BAM8-22 and ALA. Combined, our ISH and electrophysiology data suggest that some CMHs (i.e. QCs) express both MRGPRs, while others (i.e. SCs) are functionally preferentially activated by BAM8-22. Furthermore, in accordance with MRGPRD and MRGPRX1 neurons expressing TRPV1, all of these mechanosensitive C- fibers responded to noxious heat regardless of the selectivity of their responses to BAM8-22 or ALA.

\section{C-MIAs and A-fiber nociceptors are less frequently activated or unresponsive to ALA and}

\section{BAM8-22}

We also investigated whether BAM8-22 and ALA activate C-MIAs and A-fiber nociceptors which are involved in mediating itch sensation to HIS (Schmelz et al., 1997; Wooten et al., 2014) and cowhage (Ringkamp et al., 2011), respectively. None of the 6 C-MIAs tested responded to ALA. Two of 6 C-MIAs responded to BAM8-22. One of the C-MIAs had a 5 min response of 146 APs with 112 APs occurring during the first $30 \mathrm{~s}$ following the injection, and the response ending within 5 min. The other C-MIA had a 5-min response of 122 APs, 50 APs of which occurred during the first $30 \mathrm{~s}$ after injection. The response lasted for 20 minutes with a total response of 171 APs. We tested 16 mechanosensitive and 8 mechanoinsensitive A-fiber nociceptors (A-MSA and A-MIAs, respectively). Of the 16 A-MSAs, one responded to ALA (5min net response 14 APs), and another to BAM8-22 with 129 APs within the first $30 \mathrm{~s}$ after injection but no activity thereafter. Of the 8 A-MIAs, none responded to ALA or BAM8-22. 
These data suggest that A-fiber nociceptors and C-MIAs are likely minor contributors to the sensations caused by these compounds.

\section{In humans, a combination of pruritogens does not change the itch, nociceptive sensations, dysesthesias or skin reactions compared to the effects of one of the component pruritogens given alone}

In human, cowhage-induced itch and nociceptive sensations are mediated by activation of nociceptive unmyelinated and myelinated afferents that are also thought to mediate pain from noxious heat and mechanical stimuli (Johanek et al., 2008; Namer et al., 2008; Ringkamp et al., 2011; Ringkamp et al., 2013). How the sensations of itch and pain are mediated by activity in the same set of afferent nerve fibers is currently unclear, and several models have been proposed to solve this puzzle (LaMotte et al., 2014; Carstens et al., 2020). Among these, the 'spatial contrast' model posits that locally restricted activation of a small population of pruriceptive nociceptors induces itch sensation, while activation of a broader and greater number of nociceptors induces the sensation of pain (Namer et al., 2008; Namer \& Reeh, 2013; Steinhoff et al., 2019). Our present electrophysiological results indicate that ALA and BAM8-22 preferentially activate different sets of cutaneous CMHs in monkey (Figure 4). Previous studies have shown that HISinduced itch is likely mediated by C-MIAs (Schmelz et al., 1997; Wooten et al., 2014). Therefore, the 'spatial contrast' model can be tested experimentally by concurrent intradermal injections of combinations of ALA, BAM8-22 and HIS and comparison of the induced psychophysical responses to those from injection of an individual pruritogen.

The majority of subjects reported itch and nociceptive sensations after the administration of a single pruritogen and after the combinations thereof, the number of subjects reporting these sensations were comparable for the different stimuli (Figure 5A), and the number of subjects 
experiencing itch or nociceptive sensations did not change when combinations of pruritogens were applied. For the co-administration of BAM8-22 and ALA, the temporal profiles of the different sensations were visibly similar compared to those evoked by BAM8-22 or ALA alone, peaking within the first minute after application and decreasing slowly over approximately 10 minutes (Figure 5B-D). Similarly, HIS-induced itch peaked within the first minute after injection but appeared to decline more slower over 20 minutes (Figure 5E). The temporal profile of the itch sensation produced by the triple combination was similar to that of the BAM8-22+ALA combination, except that the decline of itch sensation appeared to be slower (Figure 5E). The time courses of the nociceptive sensations following HIS were similar to those of the BAM822+ALA combination and the combined application of all three pruritogens (Figure 5F-G).

To quantify and compare the responses to each of the five stimuli (three single pruritogens and two combinations) the following three measurements were obtained: The peak magnitude rating of perceived intensity, the area under the rating curve (AUC) and the duration of the ratings. For each of these measurements differences in mean response were initially statistically analyzed with a 'stimulus' (5) x 'sensory quality' (3) x 'sex' (2) RMANOVA. Only for peak ratings, but not for AUC and duration, 'sex' had a significant effect, with females giving significantly larger peak ratings than males $\left(\mathrm{F}_{(1,27)}=6.53\right.$, $\left.\mathrm{p}<0.017\right)$. However, none of the interactions with the other factors reached significance. Therefore, 'sex' was excluded from further analysis, and the RMANOVAs were re-run only with the within subject factors using Bonferroni tests for post hoc testing. For peak ratings ( $\mathrm{n}=29$ subjects), within subject factors 'sensory quality' and 'stimulus' were significant (respectively, $\mathrm{F}_{(1.67,46.67)}=12.13, \mathrm{p}<0.001$ and $\left.\mathrm{F}_{(3.31,92.80)}=3.39, \mathrm{p}<0.018\right)$. The mean peak ratings for itch and pricking/stinging were each significantly greater than those for burning ( $<<0.001$, Bonferroni test), and mean peak ratings of 
itch and pricking/stinging did not differ significantly. For the factor 'stimulus' mean peak ratings were significantly different only between ALA and BAM8-22 ( $\mathrm{p}=0.01)$, and between ALA and the combined administration of all three pruritogens $(\mathrm{p}=0.03)$. The mean peak ratings for each sensory quality and stimulus are shown in Figure 5H, but the interaction of 'stimulus' and 'sensory quality' was not significant $\left(\mathrm{F}_{(4.82,135.02)}=2.11 ; \mathrm{p}=0.07\right)$.

Comparisons of mean AUCs and durations between stimuli required subjects to have peak ratings greater than zero in response to each stimulus (because there cannot be a duration of a sensation that was absent, i.e. had zero magnitude). Seventeen subjects had non-zero ratings of all three sensory qualities in response to each stimulus. Only 'sensory quality' was significant for AUC and duration $\left(\mathrm{F}_{(1.33,21.32)}=4.89, \mathrm{p}=0.029\right.$ and $\mathrm{F}_{(1.46,23.39)}=9.18, \mathrm{p}=0.003$, respectively, RMANOVA). The mean AUC was significantly larger for itch than for burning ( $p=0.012$, Bonferroni test), and itch lasted significantly longer than burning $(\mathrm{p}<0.001$, Bonferroni test). No statistical differences were found between AUCs and durations of itch, pricking/stinging, and burning evoked by BAM8-22, ALA, HIS, and combinations thereof (Figure 5I, J).

Alternative analyses of all 25 subjects that had a nonzero rating of both itch and either of two nociceptive qualities whichever had the greater AUC revealed similar results (see Supplemental Figure 2). Thus, a combination of pruritogens did not alter the magnitude or quality of sensations over those elicited by one of its component single pruritogens. For example, increasing the number of activated mechanosensitive nociceptive afferents by combining BAM8-22 and ALA and then adding in MIAs with HIS included in the mixture did not diminish itch and increase the magnitude of nociceptive sensations.

To rule out the possibility that any one pruritogen would dominate any of the sensations when a combination was applied, we re-ran the same analysis after selecting for subjects in 
whom the lowest maximum peak itch rating to any single pruritogen was at least $50 \%$ of the highest maximum peak itch rating to any single pruritogen. Analysis of data from subjects that fulfilled this criterion $(n=11)$ did not lead to a different result than when all subjects were included. Taken together, these results suggest that intradermal injection of combinations of pruritogens does not lead to an increased sensation of itch or, alternatively, to a diminished sensation of itch with an increase in nociceptive sensations.

To test for whether any mean areas of dysesthesia, wheal and flare differed between stimuli separate RMANOVAs were performed for each type of area (Figure 6). There was a significant main effect of 'stimulus' for alloknesis $\left(\mathrm{F}_{(2.18,61.08)}=10.27, \mathrm{p}<0.001\right)$, hyperalgesia $\left(\mathrm{F}_{(2.97,83.04)}=12.79, \mathrm{p}<0.001\right)$, hyperknesis $\left(\mathrm{F}_{(3.10,86.72)}=7.14, \mathrm{p}<0.001\right)$, wheal $\left(\mathrm{F}_{(2.53,70.75)}=53.42\right.$, $\mathrm{p}<0.001)$ and flare $\left(\mathrm{F}_{(2.08,58.15)}=32.94, \mathrm{p}<0.001\right)$. The mean areas of alloknesis and hyperalgesia after HIS and the combined administration of the three pruritogens were significantly greater compared to those induced by BAM8-22, ALA, or BAM8-22+ALA (Figure 6A, see supplemental table 2 for p-values). For hyperknesis, HIS and the triple combination of pruritogens each induced significantly larger areas than either ALA or BAM8-22 alone, but not compared to the combination of BAM8-22+ALA. Notably, the combination of BAM8-22+ALA did not demonstrate any significant increase in alloknesis, hyperalgesia, or hyperknesis compared to BAM8-22 or ALA administered alone. The combination of three pruritogens also did not significantly enhance any of the dysesthesia areas compared to HIS alone.

The mean areas of wheal and flare were significantly larger in response to HIS and to the combination of all three pruritogens than in response to either BAM8-22 or ALA alone, or their combination (Figure 6B; see supplemental table 2 for p-values). Furthermore, the combination of BAM8-22+ALA did not significantly alter the areas of flare and wheal compared to each 
compound alone. Similarly, the triple combination did not cause larger areas of wheal and flare than HIS alone.

We conclude that the co-activation of different classes of CMHs or co-activation of CMHs and C-MIAs does not increase areas of dysesthesias, flare and/or wheal in comparison to the activation of distinct classes of nociceptors alone.

\section{$\underline{\text { Discussion }}$}

In our study, we investigated how itch is coded in primates, by examining the expression pattern of pruritogen receptors in human DRG, by determining physiological responses of nonhuman primate pruriceptive nociceptors, and by assessing psychophysical effects of pruritogen mixtures on itch perception in human subjects. Specifically, we explored the expression of MRGPRX1 and the physiological effects of its agonist BAM8-22, comparing these with MRGPRD expression and responses of the MRGPRD-activating pruritogen, ALA. Employing ISH, we found that MRGPRX1 and MRGPRD are predominantly co-expressed in human DRG indicating that primate and mouse pruritogen receptors have different patterns of expression. Using electrophysiology, we discovered that the MRGPRX1 and MRGPRD agonists activated predominantly cutaneous polymodal C-fibers with some differences in BAM8-22 activating almost all and ALA stimulating a smaller defined subset of C-fibers. Lastly, taking advantage of the fact that ALA, BAM8-22 and HIS differentially activate certain types of Cfibers, we investigated sensory coding of itch in humans. We observed that mixtures of these pruritogens elicit itch percepts similar to those evoked by singly applied pruritogens, suggesting that combined vs. differential fiber activation does not have a large effect in shaping the quality of sensation. 
Extensive genetic manipulation studies in mice have revealed a great deal about the receptors and sensory neurons that underlie itch in mice (Hoon, 2015; Lay \& Dong, 2020). Although less is known about the neuronal mechanisms of itch in primate, it is often assumed that they are similar to those in rodent. An example of this relates to itch induced by the BAM822 peptide, a cleavage product of proenkephalin, shown to be a pruritogen in a human psychophysical study (Sikand et al., 2011a) that also triggers scratching in mice. In rodents, BAM8-22 activates a receptor named Mrgprc (Han et al., 2002; Grazzini et al., 2004). Suggestive of a labeled-line for itch, this receptor is expressed in a select population of DRG neurons that are required for BAM8-22-induced scratching and sufficient to evoke scratching when activated (Han et al., 2013). However, primates lack the Mrgprc receptor or a differently named orthologous receptor and instead, harbor a distinct subfamily of MRGPRs, named MRGPRX (Solinski et al., 2014), i.e. clear orthologous receptors of this subfamily are absent in rodents (Zylka et al., 2003). Despite this major difference in pruritogen receptor families between primates and rodents, a striking pharmacological similarity exists between receptors in primates and mice, with MRGPRX1 in humans, like Mrgprc in mice, being activated by BAM822 (Lembo et al., 2002) and MRGPRD being activated by ALA (Liu et al., 2012; Shinohara et al., 2004). Therefore, we sought to examine the expression profile of MRGPRX1 and MRGPRD in human DRG. Similar to the expression of Mrgprc in mice, we found that MRGPRX1 is expressed in a subpopulation of human TRPV1-positive neurons. However, we found that the MRGPRD expression largely overlaps with MRGPRX1 in human DRG. This expression pattern is dramatically different from that found in mice, where Mrgprd is present in a distinct subclass of neurons that do not express Mrgprc and do not express Trpv1 (Zylka et al., 2003). These results point to primate pruriception in peripheral sensory neurons being different from that in 
mice. Previously, we reported that MRGPRX1-positive human DRG neurons express NPPB and that these cells express additional pruritogen receptors, HRH1 and IL31RA (Solinski et al., 2019a). Again, this expression pattern is different from that found in mice where $N p p b$ and Il31ra are expressed in a class of neurons distinct from those which express either Mrgprc or Mrgprd (Dong et al., 2001; Zylka et al., 2003; Zylka et al., 2005; Usoskin et al., 2015; Nguyen et al., 2017). Interestingly, $N p p b$ was shown to be necessary for the transmission of pruritic signals between primary afferents and second order spinal cord neurons in mice (Mishra \& Hoon, 2013) which in turn is suggestive of the idea that the MRGPRX1/MRGPRD/NPPB neuronal population is also involved in primate itch. Thus, our findings expand our previous knowledge on human sensory neurons and indicate that, at least at the level of the DRG soma, pruritogen receptors are expressed in a largely overlapping fashion in humans.

To complement our studies examining the expression of pruriceptive receptors in human DRG, we stimulated cutaneous afferents in monkeys with BAM8-22 and ALA and measured evoked neuronal activity, using a teased-fiber preparation. Previously, we reported that pruritogens activate a number of physiologically distinct subtypes of nerve fibers, including cutaneous CMHs, C-MIAs, as well as A-fiber nociceptors (Ringkamp \& Meyer, 2014). In addition we showed that cutaneous CMHs can be distinguished into two types based on their responses to a stepped heat stimulus, namely QC- and SC fibers (Meyer \& Campbell, 1981; Johanek et al., 2008; Wooten et al., 2014). Interestingly, ALA or BAM8-22 did not activate Afiber nociceptors, and C-MIAs were activated rarely by BAM8-22 (2/6 responsive), and not at all by ALA. Because BAM8-22 could elicit a small flare $\left(\sim 5 \mathrm{~cm}^{2}\right.$ on average $)$ in our psychophysical experiments, we cannot rule out the possibility of an indirect C-MIA activation via mast cells (Subramanian et al., 2011; Solinski et al., 2013). However, taken together, these 
findings suggest that C-MIAs and A-fiber nociceptors are not major contributors to sensations produced by ALA and BAM8-22.

In contrast, we show that CMHs are excited by intradermal injections of ALA and BAM8-22. Confirming our earlier results (Wooten et al., 2014), we found that the MRGPRD agonist ALA preferentially activated QCs, indicating that about $60 \%$ of cutaneous CMHs (nearly all QCs but only 23\% of SCs) are sensitive to ALA in monkeys. In agreement with our human expression data, BAM8-22 also activated a large proportion of QCs (72\% of QCs responded to BAM8-22), indicating that MRGPRD and MRGPRX1 are co-expressed in this functional class of cutaneous primary afferents. Surprisingly, given our expression data, we observed that BAM8-22 evoked action potentials in the SC class of CMHs (93\% of SCs responded to BAM822) that was largely unresponsive to ALA. Furthermore, BAM8-22-induced responses in SCs were about twice as large as in QCs.

Several explanations could account for this apparent discrepancy between our expression and functional data: 1) Species-specific differences, between monkeys and humans, in the DRG expression pattern of MRGPRD/MRGPRX1 might exist. 2) While ISH was used to study MRGPR expression at the neuronal soma, our physiological assay probes the functional activation of receptors in cutaneous axonal terminals. It is conceivable that mRNA expression in the soma might not be a relevant predictor for functional receptor in the periphery. 3) Potency and efficacy of GPCR agonists not only depend on the expression of the cognate receptor protein but can be modulated allosterically by binding partners of the receptor and associated proteins involved in action potential firing which could potentially be expressed differentially between QCs and SCs. Thus, we might have missed co-expression of MRGPRD and MRGPRX1 in functional studies due to cell-autonomous pharmacological differences. 
That a large proportion (54/66) of CMHs is responsive to BAM8-22 is consistent with previous findings showing that spicules of cowhage, a tropical legume, activated all tested CMHs in human (Namer et al., 2008), nearly all QC and SC mechanoheat nociceptors in monkey (Johanek et al., 2008) and that mucunain, the pruritic cysteine protease contained in these spicules activates MRGPRX1 in heterologous cells (Reddy et al., 2018). However, although about half of A-fiber nociceptors in nonhuman primate is activated by cowhage spicules (Ringkamp et al., 2011), BAM8-22 did not activate this fiber type. This suggests that cowhage responses in these fibers may be mediated through a different mechanism, for example, proteaseactivated receptors 2 and 4, which mucunain has previously been shown to activate (Reddy et al., 2008)

In nonhuman primates, we found that all mechanosensitive unmyelinated cutaneous afferents responsive to MRGPR agonists were also activated by heat, and we found previously that QCs and SCs are sensitized to heat stimuli following a mild burn injury (Wooten et al., 2014). These observations are consistent with the expression of TRPV1 in these neurons and are in agreement with the overlapping expression of TRPV1 and MRGRX1 and MRGPRD that we observed in human DRG. This is in contrast to mice in which about half of the GFP-labeled Mrgprd+ neurons in a Mrgprd reporter line were unresponsive to heat stimuli (Liu et al., 2012; Qu et al., 2014). Together, these findings show that there are key differences in the gene expression of pruriceptive receptors between mice and human and in the functional properties of cutaneous unmyelinated afferents between mice and nonhuman primate. These results will in the future be likely supplemented by the advent of single cell sequencing technologies, to determine the transcriptome of DRG neurons in human and nonhuman primates. Such studies should also 
help explain potential difference in pruriceptive sensitivity between different physiologically defined subclasses of primary afferent neurons.

In electrophysiological experiments, the peak discharge recorded from nociceptive primary afferents in our present and previous (Wooten et al., 2014) study and from pruriceptive STT neurons in the monkey (Davidson et al., 2007; Davidson et al., 2012) occurred within the first 10-30 s after cutaneous application of different pruritogens. In contrast, in psychophysical experiments, the time courses of itch and nociceptive sensations were similar for the different pruritogens with a peak intensity occurring between one and two minutes after injection. One possible explanation for this discrepancy is that the delayed peak in sensory intensity is due an increasing activation of additional nerve fibers, STT and cortical neurons when the injected pruritogen spreads over time thereby recruiting additional primary afferents or central neurons. The peak intensity of sensation would then occur when all primary afferents or central neurons within the reach of the pruritogen are activated.

Primate primary sensory afferents responsive to pruritogens are also activated by mechanical, thermal and chemical nociceptive stimuli, and activity in these afferents is thought to elicit the sensation of pain from such stimuli (Ringkamp et al., 2013). This would rule out the possibility that these primary sensory neurons constitute labeled-lines that are specifically activated only by pruritic stimuli. Consequently, several different models to explain how pruritic stimuli may be encoded, have been proposed (for review see (LaMotte et al., 2014; Carstens et al., 2020)). Among these, the itch model of 'spatial contrast' posits that a pruritic stimulus is signaled by activity in a few nociceptive afferents among other non-active fibers innervating a given skin region (i.e. spatially sparse) whereas nociceptive stimuli are encoded in the activity of many afferents (Namer et al., 2008; Schmelz, 2010; Namer \& Reeh, 2013; Steinhoff et al., 
2019). This model predicts that the sensation of itch will decrease, and the sensation of pain will increase when a larger number of nociceptive afferents is activated from a given site. Our finding of a preferential activation of distinct types of C-fiber nociceptors by ALA, BAM8-22 and HIS in monkey and reports that HIS-induced itch occurs through activation of C-MIAs in human (Schmelz et al., 1997; Wooten et al., 2014), permitted us to test a key prediction of the spatial contrast model using these itch-inducing agents in psychophysical experiments. In these experiments we used BAM8-22, ALA and HIS, each of which, when injected singly into the skin of subjects, induced itch and to a lower extent pain-like sensations of pricking/stinging and burning. Spatial contrast was then varied by co-injecting combinations of the three pruritogens, each activating different classes of primary afferents in a given skin site. We found that neither the magnitudes of itch nor nociceptive sensations changed between application of a single pruritogen and mixtures, a finding that is incongruous with the 'spatial contrast' model. This finding is also consistent with prior evidence for a lack of spatial summation or enhancement of itch when multiple cowhage spicules applied to a local area of skin did not evoke more itch than a single spicule despite presumably activating more cowhage sensitive CMHs(LaMotte et al., 2009). Similarly, we now found that increasing the number of activated nerve fibers of one type (CMHs with injection of ALA + BAM8-22) by adding those of another type (MIAs with HIS) also did not enhance the magnitude of itch.

Since the 'spatial contrast' model does not explain the results from our psychophysical experiment, what other mechanisms might be responsible for the coding of pruritic stimuli? One previously suggested solution that might explain these results is that itch- and pain-related information are separately encoded at the level of the spinal cord where pruriceptive and nociceptive STT neurons have been identified (Davidson et al., 2007; LaMotte et al., 2009). 
Input from nociceptive or pruriceptive afferents may be routed to the pruriceptive and nociceptive STT pathways through dedicated spinal interneuronal circuits. This possibility is supported by the observation that itch-like scratching behavior can be selectively blocked without reducing nociceptive behavior by Nppb deficiency (Mishra \& Hoon, 2013), by the application of antagonists of gastrin releasing peptide receptor (GRPR) (Sun \& Chen, 2007), or by eliminating GRPR-neurons (Sun et al., 2009; Sun et al., 2017). However, pruriceptive STT neurons are also responsive to noxious heat, to capsaicin, and to mechanical stimuli (Davidson et al., 2007). Thus, there must still exist a mechanism by which nociceptive afferents are able to differentially activate nociceptive and pruriceptive STT pathways. Differential activation may be achieved by the level of activity and/or the pattern of discharge that is induced by nociceptive and pruritic stimuli in nociceptive afferents. In support of this view, we have previously shown that capsaicin produced a significantly higher median discharge frequency in C-MIAs than HIS, whereas the total number of induced action potentials did not differ between the two stimuli (Wooten et al., 2014). Similarly, capsaicin produced a higher intraburst frequency discharge in HIS sensitive STT neurons than HIS (Davidson et al., 2012). In addition, activating a combination of different afferent fibers having varying tuning capabilities for particular chemical, mechanical, or thermal stimuli (e.g. QCs and SCs) and different firing patterns together may play a role in the transmission of information needed to discriminate pain from itch. Thus, while we were unable to absolutely identify the mechanism by which itch is distinguished from pain in primates, our studies suggest that the 'spatial contrast' model does not account for this process under normal conditions.

In conclusion, our study defines the expression of MRGPRX1 and MRGPRD in human DRG neurons and the electrophysiological responses of their agonists in primate cutaneous 
primary afferents. In human psychophysical studies, we observed that co-administration of different MRGPR agonists and HIS did not change overall reports of itch and nociceptive sensations. Taken together, the neuronal processing of itch differs between rodent and primate, and additional studies are needed to determine the coding mechanisms of itch and pain in human.

\section{Author contribution}

A.H.K., H.J.S. N.M.M., F.H.I., S.G.S, T.V.H., M.W., G.W., M. R. performed experiments and data analysis. R.H.L., M.H., M.R. designed and directed the experiments. A.H.K. H.J.S., M.H. R.H.L. and M.R. wrote a first draft of the manuscript which was edited by all authors. 


\section{$\underline{\text { Materials and Methods }}$}

\section{Ethics Statements}

Animal experiments were approved by the Johns Hopkins University Animal Care and Use Committee. Experiments were performed in accordance with Office of Laboratory Animal Welfare regulations and the United States Public Health Service Policy on Humane Care and Use of Laboratory Animals. Human psychophysical experiments were approved by the Yale University Internal Review Board.

\section{In situ hybridization of human DRG}

ISH of human DRG sections was performed as described previously (Solinski et al., 2019a). Briefly, postmortem DRGs from 4 different, healthy donors (see Supplemental Table 1) were obtained from Tissue For Research (www.biobankonline.com) and the NIH NeuroBioBank at the University of Maryland (Baltimore, MD). ISH staining was performed on 14-20 $\mu$ m cryosections with probes specific for hTRPV1 (\#415381), hMRGPRX1 (\#517011), and hMRGPRD (\#524871) in conjunction with the RNAscope multiplex fluorescent development kit (ACD) according to the manufacturer's instructions. Nuclei were counter-stained using DAPI. Images were collected on an Eclipse Ti (Nikon) confocal laser-scanning microscope using a 40X objective.

\section{Primate electrophysiology: Surgical Preparation}

Male and female pigtail monkeys (Macaca nemestrina, $\mathrm{n}=8$ male and 4 female) were sedated by ketamine hydrochloride (Ketaject ${ }^{\circledR}$, St. Joseph, MO; 12 mg/kg with 0.04 mg/kg atropine) followed by bolus and a continuous venous infusion of sodium pentobarbital to maintain anesthesia (Nembutal ${ }^{\circledR}$, Akorn Pharmaceuticals, Lake Forest, IL; 5 mg/kg/hr). A saline drip containing 5\% d-Glucose was continually infused to maintain hydration, and 
Cefazolin (West-Ward Pharmaceutical Corp, Eatontown, NJ; 20 mg/kg/h) was administered every 2 hrs for antibiotic prophylaxis. Body temperature was maintained by feedback-controlled heating pads $\left(\sim 38^{\circ} \mathrm{C}\right)$, and heart rate was monitored by an electrocardiogram. If heart rate increased more than $10 \%$ upon noxious stimulation, a bolus of pentobarbital was administered. Animals were intubated, paralyzed with pancuronium bromide (Sigma-Aldrich, St. Louis, MO) and ventilated at a rate to maintain an expiratory $\mathrm{pCO}_{2}$ near $40 \mathrm{mmHg}$. At the end of the experiments, animals received buprenorphine SR (0.2 mg/kg sc, ZooPharm, Laramie, WY) as prophylactic analgesic treatment before being returned to their home cage.

Radial, medial cutaneous, sural, peroneal and saphenous nerves were used for teasedfiber recordings as described previously (Campbell \& Meyer, 1983). Briefly, a given nerve was aseptically dissected under a microscope, and skin edges of the incision were sutured to a metal ring to form a pool that was filled with paraffin oil. To record neuronal activity from single nociceptive afferents, small nerve bundles were cut from the nerve proximally, teased into smaller filaments on a dissecting platform and wrapped around a silver-wire recording electrode placed above the splitting platform. Teasing of a filament continued until neuronal activity could be recorded from single nociceptive A- and C fibers. A stimulating electrode located $\sim 4 \mathrm{~cm}$ distally at the nerve trunk was used to electrically stimulate the nerve to reveal fibers on the recording electrode and to determine the conduction velocity at the nerve trunk of the afferent under study. Receptive fields (RFs) were located by applying gentle squeeze stimuli to the skin innervated by the nerve under study. RFs of nerve fibers unresponsive to such stimuli were located by electrical stimuli applied transcutaneously via a pair of ball electrodes, using a search paradigm described previously (Meyer et al., 1991; Ringkamp et al., 2001). In fibers responsive to gentle squeezing, the mechanosensitive RF was mapped with a 8.9 bar von Frey hair 
(Stoelting, Wood Dale, IL, USA), and boundaries were marked on the skin with a felt tip permanent marker. Using a slightly suprathreshold von Frey hair, the most sensitive, mechanoresponsive spot was identified within the RF and marked as the hot spot. Von Frey hairs of increasing stiffness were applied to this spot to determine the mechanical threshold of the nerve fiber. The smallest von Frey hair producing a neuronal response in 2 out of 4 applications was regarded as threshold. Units were classified as mechano-sensitive afferents (MSAs) if they were responsive to von Frey hair stimuli $<6$ bar, and mechano-insensitive afferents (MIAs) if the threshold was $>6$ bar. Transcutaneous electrical stimulation at the proximal edge of the RF was used to measure conduction latencies from the skin. Stimuli up to $100 \mathrm{~mA}$ were used, and the latency at this intensity was regarded as the conduction latency of the peripheral parent axon. Conduction distance was measured from the RF to the recording electrode using suture material placed along the presumed path of the nerve, and conduction velocities were calculated by dividing this distance by the conduction latency recorded from electrical stimulation. Fibers with a conduction velocity $<2 \mathrm{~m} / \mathrm{s}$ were classified as C- and those between $2-20 \mathrm{~m} / \mathrm{s}$ as A $\delta$-fibers. The responsiveness to heat was tested by applying a heat stimulus $\left(49^{\circ} \mathrm{C}\right.$, $3 \mathrm{~s}$ from a base temperature of $38^{\circ} \mathrm{C}$ for 3s) to the middle of the RF using a feedback-controlled carbon dioxide laser (Meyer et al., 1976). Instantaneous frequencies of responses to this stimulus were 3 point median smoothed and then used to classify C fibers as quickly (QC) or slowly (SC) responding afferents as previously described (Wooten et al., 2014). Briefly, QCs responded with a burst of discharge at the onset of the heat stimulus, and the response then quickly adapted during the temperature plateau. In contrast, SCs responded slowly, lacked a burst discharge at the response onset, and the discharge peaked during the plateau phase of the heat stimulus. Ten minutes after this 
stepped heat stimulus, a heat staircase was applied (baseline of $38^{\circ} \mathrm{C}$ for $15 \mathrm{~s}$, followed by $1^{\circ} \mathrm{C}, 1$ s increments up to $49^{\circ} \mathrm{C}$ ) to measure heat threshold.

\section{Primate electrophysiology: Chemical Stimulation}

Ten minutes after applying the heat staircase, two blocks of intradermal injections (each $10 \mu \mathrm{l}$ ) were administered at the receptive field. One block consisted of extracellular fluid (ECF, the vehicle) followed by ALA (90 $\mu$ g in ECF, Sigma-Aldrich, St Louis, MO) and another block of BAM8-18, the control peptide ( $1 \mu \mathrm{g}$ in saline, synthesized in the Keck Lab, Yale University, New Haven, CT), followed by BAM8-22 (1 $\mu \mathrm{g}$ in saline, Tocris Biosciences). Each injection (volume $10 \mu \mathrm{l}$ ) was administered with a $28 \mathrm{G} 1 / 2$ Lo-Dose syringe (Becton Dickenson, Franklin Lakes, NJ). The order of the blocks was randomized. Following each injection, neuronal activity was recorded for at least 5 minutes or until no neuronal activity was recorded for three consecutive minutes.

\section{Primate electrophysiology: Data Recording}

Action potentials were filtered, amplified, and digitized and stored for off-line data analysis using a Digital Acquisition Processor board (Microstar Laboratories Inc. Bellevue, WA) and DAPSYS software (Brian Turnquist, Bethel University, www.dapsys.net, Version 8.78). DAPSYS software was also used to control the $\mathrm{CO}_{2}$ laser stimulator, to record the skin temperature, to trigger electrical stimuli and to time intradermal injections. Action potentials (APs), trigger pulses, commands for the laser, and injection profiles were 'time-stamped' in DAPSYS and therefore allowed to relate neuronal events and stimuli applied to the receptive field.

\section{Human psychophysics: Subjects and pruritogens}


32 healthy volunteers, 15 female and 17 males were enrolled after providing written informed consent to protocols approved by the Yale University Human Investigation Committee and that met the Declaration of Helsinki Principles for human subjects. The mean age was 32.8+14.9, the median 28 years. Among these 19 were Caucasian, 8 Asian, 2 Hispanic, and 3 were more than one race. Initially, 33 subjects were recruited, one did not qualify for participation due to not feeling itch during tests with a single pruritogen and data from 3 subjects were not included in the analysis because all stimuli could not be tested due to scheduling issues.

Potential subjects were excluded from participation in the study if they reported a past medical history of cardiac, immunological, dermatological, or neurological disorders or reported taking antihistamine or analgesic medication recently.

The pruritogens, ALA (Sigma-Aldrich; $90 \mu \mathrm{g} / 10 \mu \mathrm{l} ; 100$ mM; $1.01 \mu \mathrm{mol}$ ), BAM8-22 (Tocris Bioscience; $1 \mu \mathrm{g} / 10 \mu \mathrm{l} ; 51 \mu \mathrm{M} ; 0.51 \mathrm{nmol}$ ), and HIS dihydrochloride (Sigma-Aldrich; 10 $\mu \mathrm{g} / 10 \mu \mathrm{l} ; 9 \mathrm{mM}$; $90 \mathrm{nmol}$ ), as well as a combination of 2 substances (ALA $90 \mu \mathrm{g}$ with BAM8$221 \mu \mathrm{g}$ in $10 \mu \mathrm{l}$ ) and combination of all 3 substances (ALA $90 \mu \mathrm{g}$ with BAM8-22 $1 \mu \mathrm{g}$ and HIS $10 \mu \mathrm{g}$ in $10 \mu \mathrm{l}$ ) were each prepared under sterile conditions in saline $0.9 \%$. The testing procedure was performed as previously described (LaMotte et al., 2009; Sikand et al., 2009), concentrations for injections were based on previous studies (Liu et al., 2012; Sikand et al., 2011a; Simone et al., 1991). After disinfection with an ethanol wipe subjects received a superficial intradermal injection of $10 \mu \mathrm{l}$ pruritogen solution into the volar forearm with the needle inserted approximately parallel to the skin and the tip of the needle visible through the skin (Sikand et al., 2011b). Each arm was tested maximal up to two times per session with subsequent injections made well outside the area of dysesthesias produced by a prior injection and after the sensation of the previous injection was not present anymore. The order of administration for trials was 
random and blinded for each subject. Control peptides, amino acids, and vehicles (BAM8-18, Lalanine and saline) were not administered as their evoked responses were either minimal or absent in previous human psychophysics studies (Simone et al., 1987; Sikand et al., 2011a; Liu et al., 2012).

Subjects were instructed not to scratch, or move the arm following the injection. A visual barrier was placed following the injection to cover the view on the injected arm, removing biases in ratings of sensations by observed visual changes to the skin.

\section{Human psychophysics: Ratings of Sensations after pruritogen injection}

Standardized oral instructions explaining the rating, sensations to be rated and the testing procedure were read to the subjects. Prior to testing, in an independent training session, subjects were familiarized with and instructed to rate the magnitude of sensory quality by moving a cursor to a desired position along the Labeled Magnitude Scale (gLMS) (Green et al., 1996) displayed on a computer screen. The display and recording of ratings was controlled by DAPSYS 6.0 (http://www.dapsys.net; Brian Turnquist, Bethel University, St. Paul, Minnesota).

The computer converted the chosen position to a numerical score (not visible to the subject) between ' 0 ' at the bottom of the line to ' 100 ' at the top. The scale was labeled with a quasilogarithmical distribution of descriptive labels: ”no sensation” (located at a numerical position of 0), ”barely detectable” (1), ”weak” (6), ”moderate” (17), ”strong” (35), ”very strong” (53),

”strongest imaginable sensation of any kind” (100). Each rating value on the gLMS corresponded with the maximum sensation felt by the individual within the last 30 second interval for each of 3 distinct sensation categories: “itching”, “pricking/stinging”, and "burning” (LaMotte et al., 2009). Itch was defined as a sensation that evokes a desire to scratch. Pricking/stinging was defined as a nociceptive sensation that was sharp and well-localized, either 
intermittent like a needle prick or continuous like an insect sting. Burning was defined as a nociceptive sensation most often associated with sunburns or thermal burns, but also with skin abrasions, strong cold, and/or chemical irritants. Instructions also distinguished the above sensations from others and subjects were specifically asked to rate the magnitude of nociceptive ('pain-like”) sensations regardless of whether or not overt pain occurred. Subjects were asked to ignore the initial prick of the needle entering the skin immediately after the injection. Subjects were prompted to rate levels of sensations experienced only in and surrounding the area of the application of the pruritogen every 30 seconds for a minimum of 5 and a maximum of 20 minutes or until each sensory quality received three successive ratings of zero magnitude.

\section{Human psychophysics: Skin reactions and areas of dysesthesias}

After the conclusion of the sensory ratings, the visual barrier was removed and superficial changes to the skin's appearance were marked on the skin. These could include a wheal (raised edematous region), and/or a "flare” herein broadly defined to include visible erythema that appears confined to the immediate vicinity of the injection site or extends to a larger area surrounding the site.

Next, subjects were tested for the presence of mechanically evoked dysesthesias surrounding the injection site. Mechanical testing proceeded in a radial manner beginning on the outer edge of the arm and moving inward nearing the center of the injection. Alloknesis was denoted as an area within which itch was evoked by light stroking with a cotton swab. Hyperalgesia and hyperknesis were defined as enhanced pricking pain and itch evoked by von Frey type filaments with tip diameters (and exerting bending forces) of $200 \mu \mathrm{m}(80 \mathrm{mN})$ and 50 $\mu \mathrm{m}(20 \mathrm{mN})$, respectively, each applied for $1 \mathrm{sec}$. The borders of each area were marked with a felt tip pen. 
The marked areas of changes in skin appearance and dysesthesia were photographed with a scale for further offline analysis and measurement using ImageJ (version 1.51, NIH, Bethesda, Maryland, USA).

\section{Data analysis}

In situ hybridization: For each double-labeling experiment, sections from 4 different tissue donors were stained. To avoid bias, complete DRG sections were imaged and individual images stitched together using NIS elements (Nikon) to generate an image of the whole DRG. Initially, fluorescent channels of the stitched images were handled separately and visually analyzed for neuronal expression of stained markers in ImageJ. A neuron, defined by a broad DAPI- positive neuronal nucleus and/or a dense array of surrounding DAPI-positive satellite glia nuclei, was manually counted as positive only when $>5$ puncta per cell were present. Post counting individual fluorescent channels, the channels were merged and potential co-expression of marker genes was assessed permitting blind quantification of co-expression. Data is expressed as mean percentage \pm and standard error of the mean calculated across the 4 tissue donors and as aggregated number of positive neurons from all 4 tissue donors.

Primate electrophysiology: For each injection, spontaneous activity during the one minute of baseline recording prior to the injection was prorated for the duration of the recorded response and subtracted from the total number of action potentials recorded following the injection. Responses from the control compound were subtracted to calculate a net response to the pruritogen. A unit was classified as being responsive to a pruritogen, if the net response was $\geq 10$ APs/5 min. Data were analyzed with parametric (ANOVA) and non-parametric $\left(\chi^{2}\right)$ tests where appropriate (see text for details). 
Human psychophysics: For each subject's ratings of each sensory quality the following parameters were obtained: 1) the peak rating (highest rating of magnitude), 2) the duration of the sensation the duration of sensation (elapsed time between the onset of non-zero ratings and the first rating of 0 rating after the sensation disappeared), and 3) the area under the rating curve over time (AUC) for the duration of the sensation. For each of the three measurements and each area of any dysesthesia, flare or wheal, statistical comparisons of means in response to each single pruritogen and the two combinations for pruritogens were analyzed using within subject repeated measures analyses of variance (RMANOVAs see main text and supplementary data for details). Since these data failed the test for sphericity, the Greenhouse-Geisser correction was applied. The Bonferroni test was used for post hoc testing. All electrophysiological and psychophysical data were analyzed with Statistica 13 (TIBCO Software Inc., Palo Alto, CA). A value of p < 0.05 was considered as statistically significant. Figures were prepared with SigmaPlot 10 (Systat Software Inc, San Jose, CA), and, when necessary, modified with CorelDraw (version 9.439 or 2020, Corel Corporation, Ottawa, Canda) and produced in their final version with Adobe Illustrator. Data are presented as mean \pm SEM. 


\section{References}

Campbell, J. N. \& Meyer, R. A. (1983). Sensitization of unmyelinated nociceptive afferents in the monkey varies with skin type. Journal of Neurophysiology, 49, 98-110.

Carstens, E., Follansbee, T., \& Iodi, C. M. (2020). The Challenge of Basic Itch Research. Acta Derm.Venereol., 100, adv00023.

Davidson, S., Zhang, X., Khasabov, S. G., Moser, H. R., Honda, C. N., Simone, D. A. et al. (2012). Pruriceptive spinothalamic tract neurons: physiological properties and projection targets in the primate. Journal of Neurophysiology, 108, 1711-1723.

Davidson, S., Zhang, X., Yoon, C. H., Khasabov, S. G., Simone, D. A., \& Giesler, G. J., Jr. (2007). The itch-producing agents histamine and cowhage activate separate populations of primate spinothalamic tract neurons. Journal of Neuroscience, 27, 10007-10014.

Dong, X., Han, S., Zylka, M. J., Simon, M. I., \& Anderson, D. J. (2001). A diverse family of GPCRs expressed in specific subsets of nociceptive sensory neurons. Cell, 106, 619-632.

Grazzini, E., Puma, C., Roy, M. O., Yu, X. H., O'Donnell, D., Schmidt, R. et al. (2004). Sensory neuron-specific receptor activation elicits central and peripheral nociceptive effects in rats. Proc.Natl.Acad.Sci.U.S.A, 101, 7175-7180.

Green, B. G., Dalton, P., Cowart, B., Shaffer, G., Rankin, K., \& Higgins, J. (1996). Evaluating the 'Labeled Magnitude Scale' for measuring sensations of taste and smell. Chem.Senses, 21, 323-334. 
Han, L., Ma, C., Liu, Q., Weng, H. J., Cui, Y., Tang, Z. et al. (2013). A subpopulation of nociceptors specifically linked to itch. Nat.Neurosci, 16, 174-182.

Han, S. K., Dong, X., Hwang, J. I., Zylka, M. J., Anderson, D. J., \& Simon, M. I. (2002). Orphan G protein-coupled receptors MrgA1 and MrgC11 are distinctively activated by RFamide-related peptides through the Galpha q/11 pathway. Proc.Natl.Acad.Sci.U.S.A, 99, 14740-14745.

Hoon, M. A. (2015). Molecular dissection of itch. Current Opinion in Neurobiology, 34, 61-66.

Johanek, L. M., Meyer, R. A., Friedman, R. M., Greenquist, K. W., Shim, B., Borzan, J. et al. (2008). A role for polymodal C-fiber afferents in nonhistaminergic itch. Journal of Neuroscience, 28, 7659-7669.

Johanek, L. M., Meyer, R. A., Hartke, T., Hobelmann, J. G., Maine, D. N., LaMotte, R. H. et al. (2007). Psychophysical and physiological evidence for parallel afferent pathways mediating the sensation of itch. Journal of Neuroscience, 27, 7490-7497.

LaMotte, R. H., Dong, X., \& Ringkamp, M. (2014). Sensory neurons and circuits mediating itch. Nat.Rev.Neurosci., 15, 19-31.

LaMotte, R. H., Shimada, S. G., Green, B. G., \& Zelterman, D. (2009). Pruritic and nociceptive sensations and dysesthesias from a spicule of cowhage. Journal of Neurophysiology, 101, 1430-1443.

Lay, M. \& Dong, X. (2020). Neural Mechanisms of Itch. Annu.Rev.Neurosci., 43, 187-205. 
Lembo, P. M., Grazzini, E., Groblewski, T., O'Donnell, D., Roy, M. O., Zhang, J. et al. (2002).

Proenkephalin A gene products activate a new family of sensory neuron--specific GPCRs. Nat.Neurosci, 5, 201-209.

Liu, Q., Sikand, P., Ma, C., Tang, Z., Han, L., Li, Z. et al. (2012). Mechanisms of itch evoked by beta-alanine. Journal of Neuroscience, 32, 14532-14537.

Liu, Q., Tang, Z., Surdenikova, L., Kim, S., Patel, K. N., Kim, A. et al. (2009). Sensory neuronspecific GPCR Mrgprs are itch receptors mediating chloroquine-induced pruritus. Cell, 139, 1353-1365.

Meyer, R. A. \& Campbell, J. N. (1981). Evidence for two distinct classes of unmyelinated nociceptive afferents in monkey. Brain Research, 224, 149-152.

Meyer, R. A., Davis, K. D., Cohen, R. H., Treede, R.-D., \& Campbell, J. N. (1991). Mechanically insensitive afferents (MIAs) in cutaneous nerves of monkey. Brain Research, 561, 252-261.

Meyer, R. A., Walker, R. E., \& Mountcastle, V. B. (1976). A laser stimulator for the study of cutaneous thermal pain sensation. IEEE Trans.Biomed.Eng., 23, 54-60.

Mishra, S. K. \& Hoon, M. A. (2013). The cells and circuitry for itch responses in mice. Science, 340, 968-971.

Namer, B., Carr, R., Johanek, L. M., Schmelz, M., Handwerker, H. O., \& Ringkamp, M. (2008). Separate peripheral pathways for pruritus in man. Journal of Neurophysiology, 100, 2062-2069. 
Namer, B. \& Reeh, P. (2013). Scratching an itch. Nat.Neurosci., 16, 117-118.

Nguyen, M. Q., Wu, Y., Bonilla, L. S., von Buchholtz, L. J., \& Ryba, N. J. P. (2017). Diversity amongst trigeminal neurons revealed by high throughput single cell sequencing. PLOS ONE, 12, e0185543.

Qu, L., Fan, N., Ma, C., Wang, T., Han, L., Fu, K. et al. (2014). Enhanced excitability of MRGPRA3- and MRGPRD-positive nociceptors in a model of inflammatory itch and pain. Brain, 137, 1039-1050.

Rau, K. K., McIlwrath, S. L., Wang, H., Lawson, J. J., Jankowski, M. P., Zylka, M. J. et al. (2009). MrgprD enhances excitability in specific populations of cutaneous murine polymodal nociceptors. Journal of Neuroscience, 29, 8612-8619.

Reddy, V. B., Azimi, E., Chu, L., \& Lerner, E. A. (2018). Mas-Related G-Protein Coupled Receptors and Cowhage-Induced Itch. J.Invest Dermatol., 138, 461-464.

Reddy, V. B., Iuga, A. O., Shimada, S. G., LaMotte, R. H., \& Lerner, E. A. (2008). Cowhageevoked itch is mediated by a novel cysteine protease: a ligand of protease-activated receptors. Journal of Neuroscience, 28, 4331-4335.

Ringkamp, M. \& Meyer, R. (2014). Pruriceptors. In E.Carstens \& T. Akiyama (Eds.), Itch: Mechanisms and Treatment ( Boca Raton (FL): CRC Press/Taylor \& Francis.

Ringkamp, M., Peng, Y. B., Wu, G., Hartke, T. V., Campbell, J. N., \& Meyer, R. A. (2001). Capsaicin responses in heat-sensitive and heat-insensitive A-fiber nociceptors. Journal of Neuroscience, 21, 4460-4468. 
Ringkamp, M., Raja, S. N., Campbell, J. N., \& Meyer, R. A. (2013). Peripheral mechanisms of cutaneous nociception. In S.B.McMahon, M. Koltzenburg, I. Tracey, \& D. Turk (Eds.), Textbook of Pain (6 ed., pp. 1-30). Philadelphia: Elsevier Saunders.

Ringkamp, M., Schepers, R. J., Shimada, S. G., Johanek, L. M., Hartke, T. V., Borzan, J. et al. (2011). A role for nociceptive, myelinated nerve fibers in itch sensation. Journal of Neuroscience, 31, 14841-14849.

Schmelz, M. (2010). Itch and pain. Neurosci Biobehav.Rev., 34, 171-176.

Schmelz, M., Schmidt, R., Bickel, A., Handwerker, H. O., \& Torebjörk, H. E. (1997). Specific C-receptors for itch in human skin. Journal of Neuroscience, 17, 8003-8008.

Shinohara, T., Harada, M., Ogi, K., Maruyama, M., Fujii, R., Tanaka, H. et al. (2004). Identification of a $\mathrm{G}$ protein-coupled receptor specifically responsive to beta-alanine. J.Biol.Chem., 279, 23559-23564.

Sikand, P., Dong, X., \& LaMotte, R. H. (2011a). BAM8-22 peptide produces itch and nociceptive sensations in humans independent of histamine release. Journal of Neuroscience, 31, 7563-7567.

Sikand, P., Shimada, S. G., Green, B. G., \& LaMotte, R. H. (2009). Similar itch and nociceptive sensations evoked by punctate cutaneous application of capsaicin, histamine and cowhage. Pain, 144, 66-75. 
Sikand, P., Shimada, S. G., Green, B. G., \& LaMotte, R. H. (2011b). Sensory responses to injection and punctate application of capsaicin and histamine to the skin. Pain, 152, 2485-2494.

Simone, D. A., Alreja, M., \& LaMotte, R. H. (1991). Psychophysical studies of the itch sensation and itchy skin ("Alloknesis") produced by intracutaneous injection of histamine. Somatosensory and Motor Research, 8, 271-279.

Simone, D. A., Ngeow, J. Y., Whitehouse, J., Becerra-Cabal, L., Putterman, G. J., \& LaMotte, R. H. (1987). The magnitude and duration of itch produced by intracutaneous injections of histamine. Somatosens.Res, 5, 81-92.

Solinski, H. J., Dranchak, P., Oliphant, E., Gu, X., Earnest, T. W., Braisted, J. et al. (2019a). Inhibition of natriuretic peptide receptor 1 reduces itch in mice. Sci.Transl.Med., 11.

Solinski, H. J., Gudermann, T., \& Breit, A. (2014). Pharmacology and signaling of MAS-related G protein-coupled receptors. Pharmacol.Rev., 66, 570-597.

Solinski, H. J., Kriegbaum, M. C., Tseng, P. Y., Earnest, T. W., Gu, X., Barik, A. et al. (2019b). Nppb Neurons Are Sensors of Mast Cell-Induced Itch. Cell Rep., 26, 3561-3573.

Solinski, H. J., Petermann, F., Rothe, K., Boekhoff, I., Gudermann, T., \& Breit, A. (2013). Human Mas-related G protein-coupled receptors-X1 induce chemokine receptor 2 expression in rat dorsal root ganglia neurons and release of chemokine ligand 2 from the human LAD-2 mast cell line. PLoS.ONE., 8, e58756. 
Steinhoff, M., Oaklander, A. L., Szabo, I. L., Stander, S., \& Schmelz, M. (2019). Neuropathic itch. Pain, 160 Suppl 1, S11-S16.

Subramanian, H., Kashem, S. W., Collington, S. J., Qu, H., Lambris, J. D., \& Ali, H. (2011). PMX-53 as a dual CD88 antagonist and an agonist for Mas-related gene 2 (MrgX2) in human mast cells. Mol.Pharmacol., 79, 1005-1013.

Sun, S., Xu, Q., Guo, C., Guan, Y., Liu, Q., \& Dong, X. (2017). Leaky Gate Model: IntensityDependent Coding of Pain and Itch in the Spinal Cord. Neuron, 93, 840-853.

Sun, Y. G. \& Chen, Z. F. (2007). A gastrin-releasing peptide receptor mediates the itch sensation in the spinal cord. Nature, 448, 700-703.

Sun, Y. G., Zhao, Z. Q., Meng, X. L., Yin, J., Liu, X. Y., \& Chen, Z. F. (2009). Cellular basis of itch sensation. Science, 325, 1531-1534.

Usoskin, D., Furlan, A., Islam, S., Abdo, H., Lonnerberg, P., Lou, D. et al. (2015). Unbiased classification of sensory neuron types by large-scale single-cell RNA sequencing. Nature Neuroscience, 18, 145-153.

Wooten, M., Weng, H. J., Hartke, T. V., Borzan, J., Klein, A. H., Turnquist, B. et al. (2014). Three functionally distinct classes of C-fibre nociceptors in primates. Nature Communications, 5, 4122.

Zylka, M. J., Dong, X., Southwell, A. L., \& Anderson, D. J. (2003). Atypical expansion in mice of the sensory neuron-specific Mrg G protein-coupled receptor family. Proc.Natl.Acad.Sci.U.S.A, 100, 10043-10048. 
bioRxiv preprint doi: https://doi.org/10.1101/2020.11.04.368597; this version posted November 4, 2020. The copyright holder for this preprint

(which was not certified by peer review) is the author/funder, who has granted bioRxiv a license to display the preprint in perpetuity. It is made available under aCC-BY 4.0 International license.

Zylka, M. J., Rice, F. L., \& Anderson, D. J. (2005). Topographically distinct epidermal nociceptive circuits revealed by axonal tracers targeted to Mrgprd. Neuron, 45, 17-25. 


\section{$\underline{\text { Figures }}$}
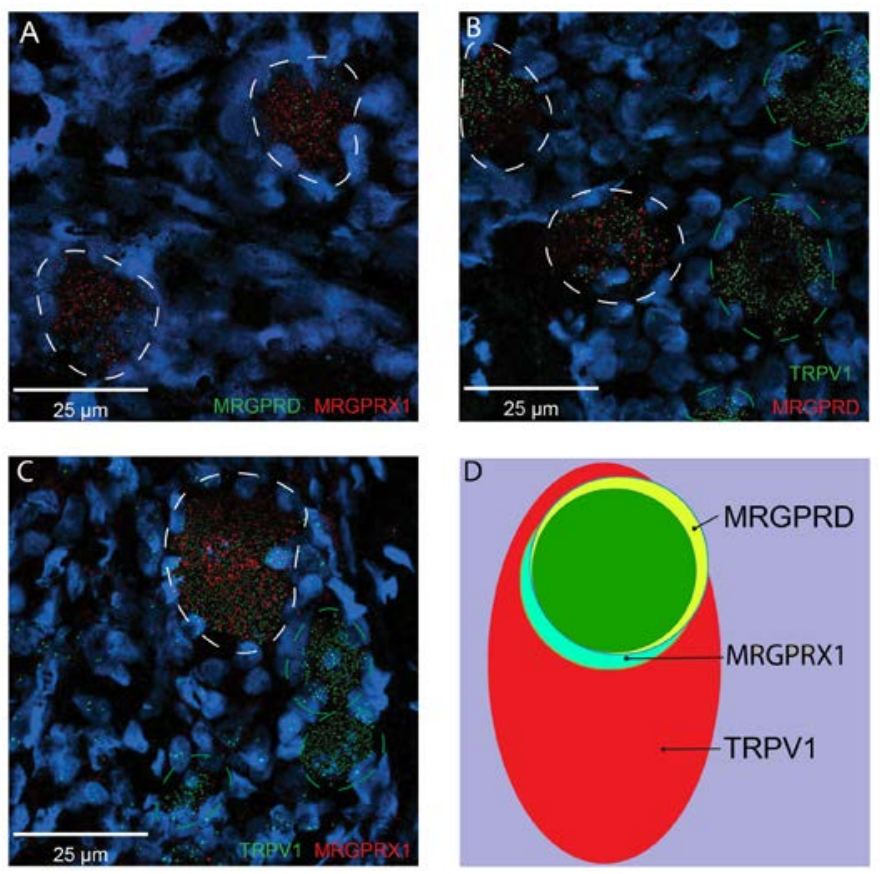

Figure 1. MRGPRX1 and MRGPRD are co-expressed in TRPV1 expressing human DRG neurons.

(A-C) Representative double ISH images of a field of human DRG with neurons stained for MRGPRD (A, green; B, red), MRGPRX1 (A and C, red) and TRPV1 (B and C, green).

Double-positive and single-positive neurons are outlined with white and green dashed lines, respectively. DAPI counterstain is displayed in blue. (D) Venn diagram summarizing the relative expression overlap of MRGPRD (yellow), MRGPRX1 (blue) and TRPV1 (red) in human DRG. Note that MRGPRD and MRGPRX1 are expressed in a largely overlapping population (green, i.e. MRGPRD + MRGPRX1/MRGPRD = $89.6 \pm 1.5 \%$; MRGPRX1 + MRGPRD/MRGPRX1 $=93.9 \pm 2.0 \%$ ) in about 1/3 of all TRPV1-positive neurons (MRGPRD + TRPV1/TRPV1 = $36.5 \pm$ 4.2\%; MRGPRX1 + TRPV1/TRPV1 = $32.5 \pm 3.7 \%$ ). Expression analysis for all three markers was performed in DRG tissue from 4 individuals and data are stated as mean \pm SEM. Green filled area indicates the overlap in expression of MRGPRD and MRGPRX1. 

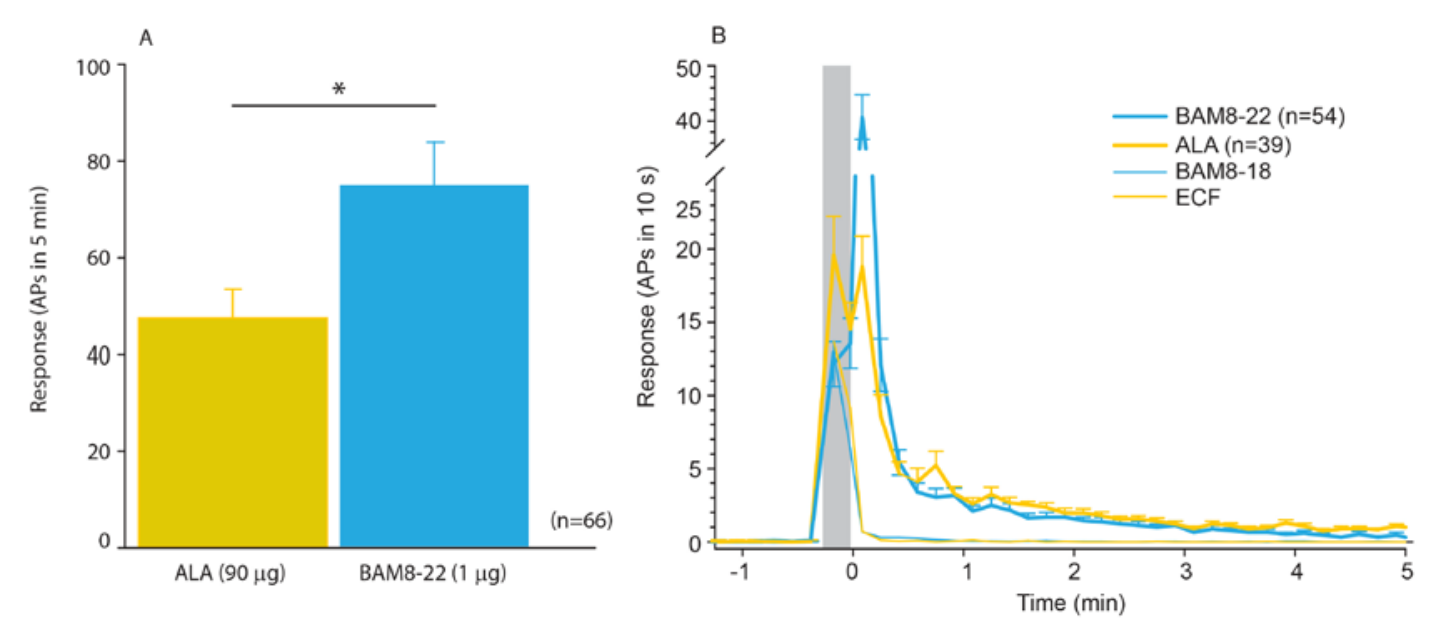

Figure 2. CMHs respond to BAM8-22 more vigorously than to ALA but with a similar time courses. (A) The average evoked response of all CMHs to BAM8-22 (blue), was significantly larger than that to ALA (yellow) injection (paired t-test: $\mathrm{t}_{(65)}=-2.142, \mathrm{p}=$ 0.0359). (B) The time course of action potential activity (plotted as number of APs in $10 \mathrm{~s}$ bins) was similar after BAM8-22 and ALA injection, except for the greater response to BAM8-22 within the first $10 \mathrm{~s}$ following injection. The average responses of the same populations to vehicle (ECF and BAM8-18) are graphed with thinner lines. Error bars represent standard error of the mean (SEM). Grey box marks time of needle insertion and injection. 

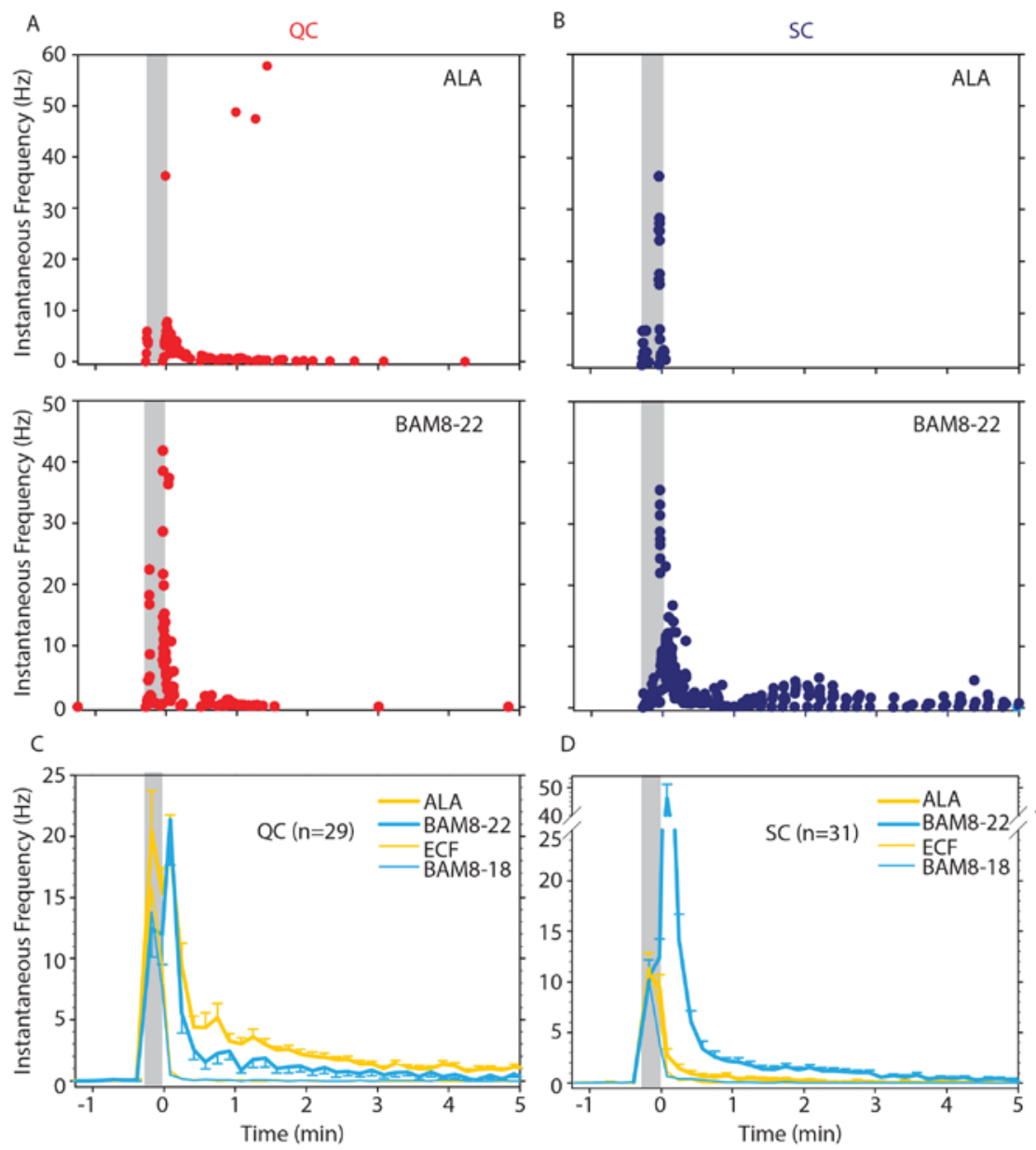

Figure 3. CMH subpopulations, QCs and SCs, exhibit differences in the magnitude and time course of their response to ALA and BAM8-22. Examples of responses in (A) of a QC fiber and (B) a SC fiber to ALA (top panels) and each to BAM8-22 (bottom panels). Responses to ALA and BAM8-22 are from the same fiber. The instantaneous frequency of each action potential is plotted vs the time of its occurrence. The time course of neuronal activity induced by ALA, BAM8-22 and vehicle controls in the population of (C) QC fibers and (D) SC fibers. The average number of action potentials recorded over $10 \mathrm{~s}$ intervals during the 5 min observation period following injection is plotted. In QC fibers, ALA and BAM8-22 produced marked excitation, whereas in SC fibers, only BAM8-22 produced long-lasting activity. Error bars represent standard error of the mean $( \pm \mathrm{SEM})$. Grey boxes mark time of needle insertion and injection. 

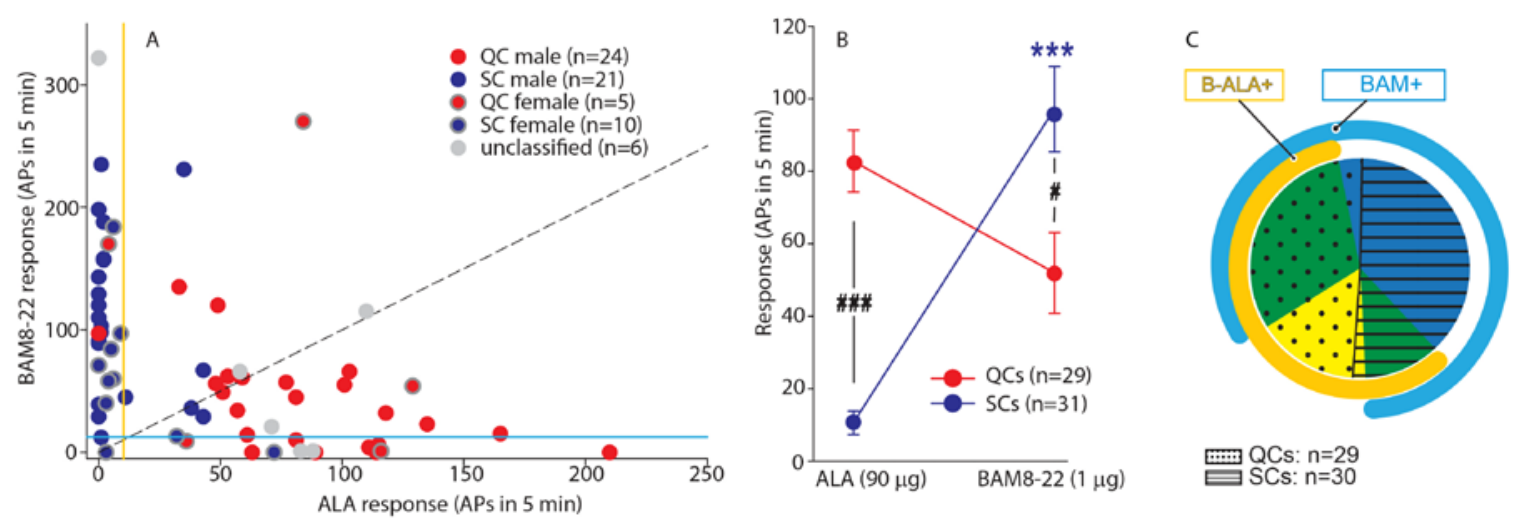

Figure 4. QCs and SCs from both male and female monkeys differ in sensitivity and incidence of activation by ALA and BAM8-22. For each C-fiber, the response to BAM822 was plotted against its response to ALA. Solid red and blue circles indicate QC- and SC fibers, respectively, recorded from male animals. Red and blue circles with a grey border indicate data obtained from female animals. Solid grey circles indicate data from $\mathrm{C}$ fibers with unclassified heat response. The diagonal line indicates equal response to both compounds. Vertical and horizontal lines indicate the 'threshold' ( $\geq 10$ APs) for an afferent to be counted as being responsive to an agonist (yellow and blue, respectively for responses to ALA and BAM8-22). (B) Population responses of QCs and SCs to ALA and BAM8-22. In SCs, intradermal injection of BAM8-22 produces a significantly greater response than ALA (***, $\mathrm{p}<0.0001)$. The response to ALA was significantly larger in QCs than SCs (\#\#\#, $\mathrm{p}<0.0001$ ), whereas the response to BAM8-22 was significantly larger in SCs than QCs (\#, $\mathrm{p}=0.002)$. Data were analyzed with RMANOVA with 'fiber type' as an in between subject factor and 'pruritogen' as within subject factor ('fiber type' $x$ 'pruritogen': $F_{(1,58)}=29,55$; $\mathrm{p}<0.0001$ ), followed by Scheffe-test for post hoc analysis. (C) ALA activated 27/29 QCs and 7/31 SCs. BAM8-22 activated 21/29 QCs and 29/31 SCs. The occurrence of QCs and SCs responding to ALA only (8 and 1 afferents, respectively) was fairly rare. The number of QCs responding only to BAM8-22 (2 units) was also small, whereas the majority of SCs (23/31) only responded to BAM8-22. Of the 31 SCs, one did not respond to either agonist. 19 QCs and 6 SCs responded to both, ALA and BAM8-22. The incidence of responsiveness to BAM8-22 and ALA was significantly different $\left(\mathrm{X}^{2}{ }_{(1)}=8.19, \mathrm{p}=0.0042\right)$. 

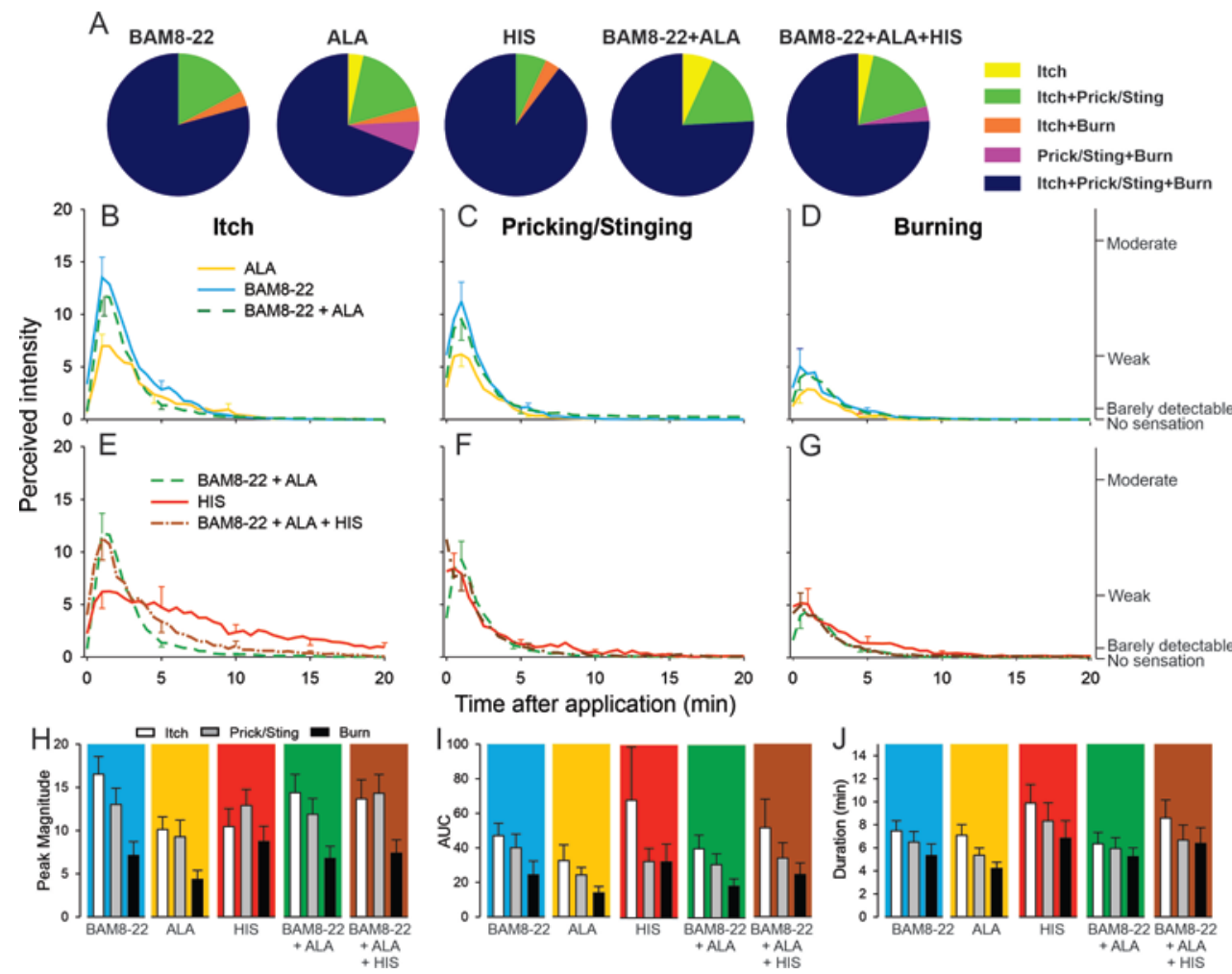

Figure 5. In humans, co-injection of ALA and BAM8-22 with or without HIS does not change the itch or nociceptive sensations compared to the effects of one component pruritogen given alone. (A) The majority of subjects $(n=29)$ reported itch, pricking/stinging and burning sensations after administration of each of the pruritogens or their combinations. (B) Magnitude of itch, (C) stinging/pricking, and (D) burning sensations evoked by BAM8-22, ALA, and a combination of BAM8-22 and ALA are plotted for successive $30 \mathrm{~s}$ intervals after injection averaged across all 29 subjects. (E) Magnitude of itch, $(F)$ stinging/pricking, and $(G)$ burning sensations evoked by HIS, a combination of BAM8-22 and ALA, and a combination of BAM8-22, ALA, and HIS. For clarity, the SEM is plotted only every 5 min starting with the peak rating for each quality. On the right vertical axis, the locations of three verbal descriptors are shown in correspondence with the ratings of perceived intensity indicated on the left vertical axis (see Materials and Methods). (H) Peak magnitude of perceived sensation intensity $(n=29)$, (I) computed area under the curve $(n=17)$ or $(J)$ duration of itch, stinging/pricking, or burn ratings $(n=17)$ were each not significantly different for the 5 different stimuli, i.e. three single pruritogens and two combinations (RMANOVA, see text for further details). For panels I and J, subjects $(n=12)$ without a measurable AUC or duration of any sensation during the time course were not included in the analysis and therefore only 17 subjects were used for within-subject analysis and post hoc comparisons. Data represent mean \pm standard error of the mean (SEM). 

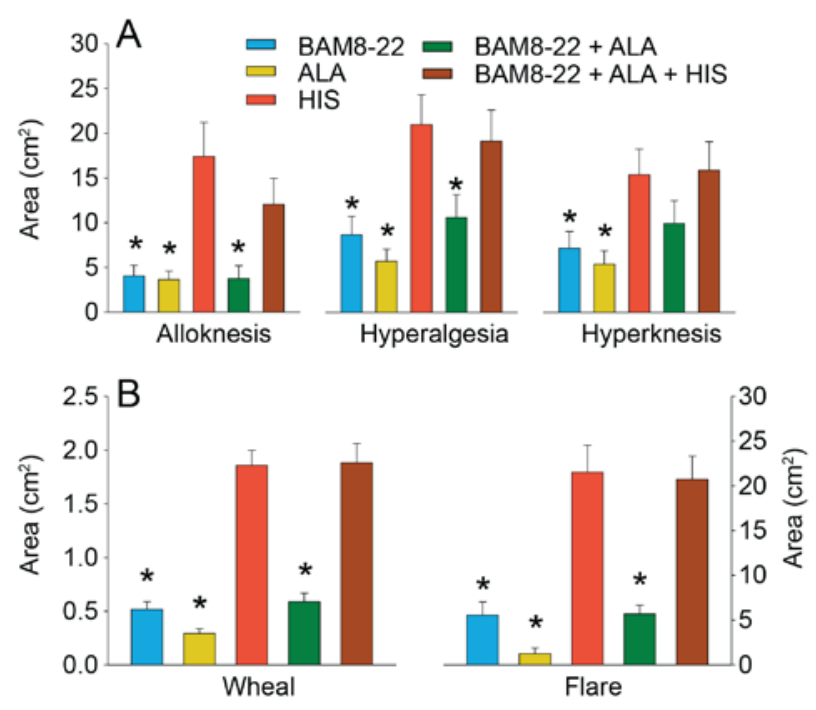

Figure 6. In humans, co-injection of ALA and BAM8-22 with or without HIS does not change the areas of dysesthesia or skin reactions compared to the effects of one of the component pruritogens given alone. (A) Areas of alloknesis, hyperalgesia, and hyperknesis and (B) Areas of wheal and flare produced after injection of each pruritogen (BAM8-22, ALA, HIS and combination of pruritogens. Data were analyzed with RMANOVAs. Greenhouse-Gessier corrections were made for all analyses to correct for non-sphericity followed by Bonferroni post hoc tests (see main text and Supplemental Table 2 for p values). *: indicates significant differences compared to HIS or BAM8$22+$ ALA+HIS. Data from all subjects $(n=29)$ were included in the analysis. Data represent mean \pm standard error of the mean (SEM). 


\section{Supplemental Figures}
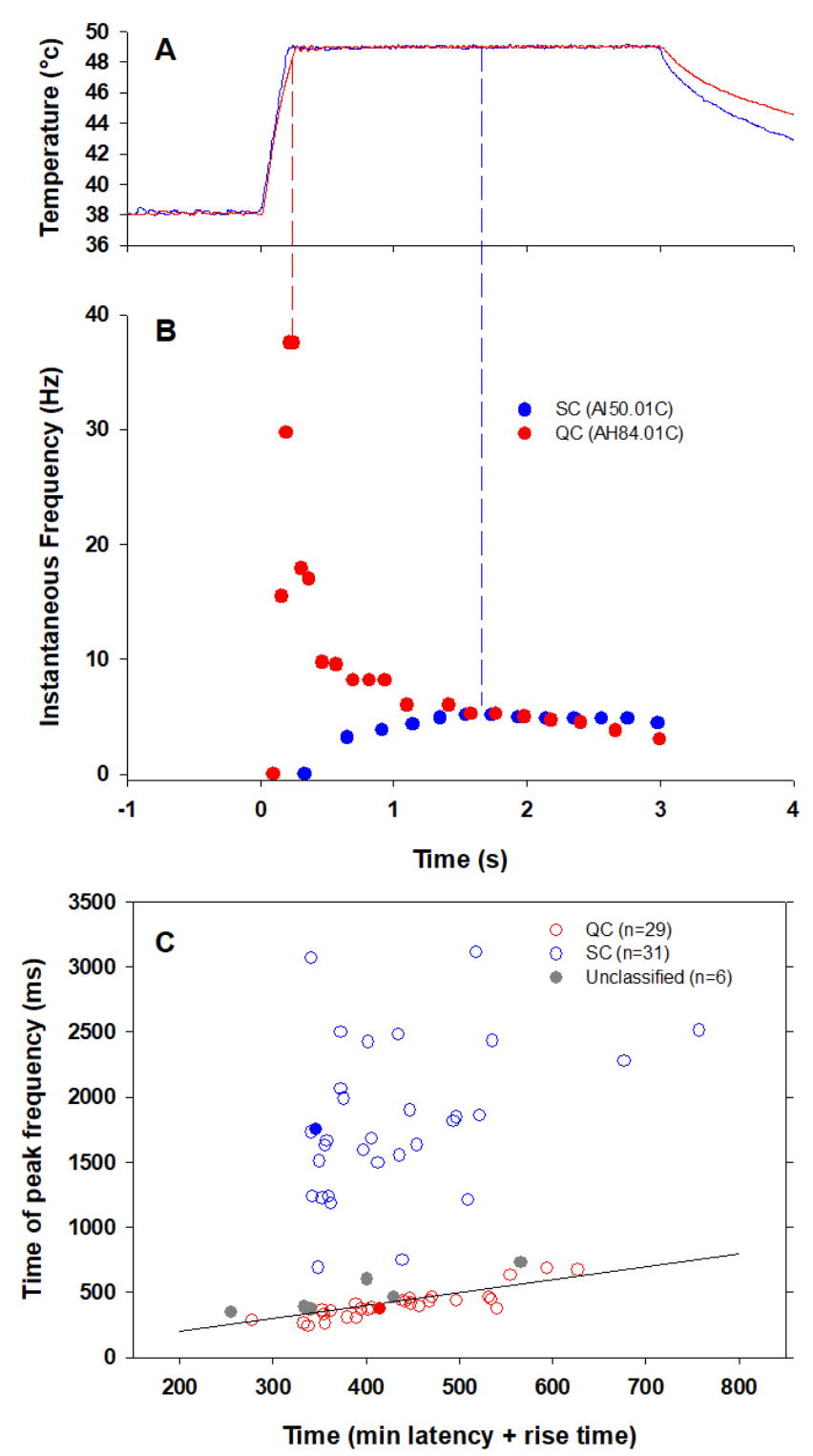

Supplemental Figure 1. Examples of QC and SC heat responses. (A) Temperature waveform of the $\mathrm{CO}_{2}$ laser-evoked heat stimulus. The skin was first pre-heated to $38^{\circ} \mathrm{C}, 3 \mathrm{sec}$ baseline temperature and then rapidly raised (rise time $\sim 200 \mathrm{msec}$ ) to $49^{\circ} \mathrm{C}$ for $3 \mathrm{sec}$. (B) Specimen recording showing the response of an individual QC fiber (red circles) and a SC fiber (blue circles) to the laser-heat stimulus described in (A). The instantaneous discharge frequency is plotted versus time. Each dot represents the occurrence of an action potential. The response in the QC fiber starts during the temperature rise and reaches peak frequency at the end of the ramp (red dashed line). The SC fiber reached the peak instantaneous discharge frequency during the plateau phase of the heat stimulus (blue dashed line). (C) The time of peak discharge for each fiber is plotted against the sum of stimulus rise time + the minimal AP conduction time as measured in response to transcutaneous electrical stimulation from the proximal edge of the RF. 
Data points above the line of equality correspond to fibers in which the peak discharge occurred in the plateau phase of the heat stimulus (SCs, blue circles). Data points falling below the line of equality are from those fibers whose peak discharge occurred during the rising phase of the heat stimulus (QCs, red circles). The filled circles represent the data from the specimen recordings shown in (B) and also of the specimen responses to ALA and BAM8-22 shown in Figure 3. Grey circles indicate data from fibers that were unclassified. 

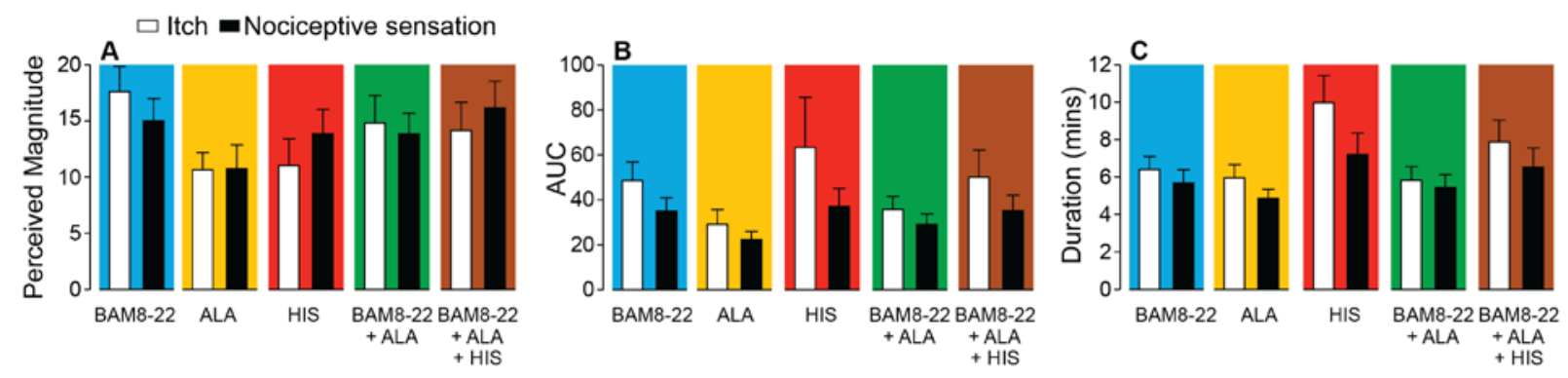

Supplemental Figure 2. In humans, co-injection of ALA and BAM8-22 with or without HIS does not change the itch or nociceptive sensation compared to the effects of one of the component pruritogens given alone. Data are plotted in the same format as Fig. $5 \mathrm{H}-\mathrm{J}$ for the 25 subjects that, in response to each single pruritogen, reported a greater than zero peak magnitude of both itch and either type of nociceptive sensation (whichever elicited the greater AUC). This increased the sample size from $n=17$ in Fig. 5I-J to $n=25$ by including the data for eight additional subjects that always felt at least one type of nociceptive sensation but not always both. A 5 'stimulus' x 2 'sensory quality' RMANOVA was performed for peak magnitude, AUC and duration. For peak magnitude, only 'stimulus' had a significant effect $\left(F_{(4,96)}=2.85, p<0.03\right)$ with BAM8-22 producing a higher peak magnitude of sensation than ALA ( $\mathrm{p}<0.034$, Bonferroni test). The interaction between 'stimulus' and 'sensory quality' was not significant $\left(\mathrm{F}_{(4,96)}=1.89\right.$, $\mathrm{p}=0.12)$. For AUC, only 'sensory quality' was significant $\left(\mathrm{F}_{(1,24.0)}=5.25, \mathrm{p}<0.032\right)$, with the mean AUC for itch being significantly larger than that for nociceptive sensation $(\mathrm{p}<0.032$, Bonferroni test). The interaction between 'stimulus' and 'sensory quality' was not significant $\left(\mathrm{F}_{(1.7,42.2)}=0.91\right.$, $\mathrm{p}=0.40)$. For duration, 'stimulus' and 'sensory quality' were each significant $\left(\mathrm{F}_{(2.75,66.0)}=4.25\right.$, $\mathrm{p}=0.010$ and $\mathrm{F}_{(1,24.0)}=13.03, \mathrm{p}=0.001$, respectively), but their interaction was not $\left(\mathrm{F}_{(2.78,66,8)}=2.1\right.$, $\mathrm{p}=0.11$ ). HIS-induced sensations lasted significantly longer than those induced by ALA or by BAM8-22+ALA ( $<<0.01$ and $p<0.05$, respectively). Furthermore, the duration of itch was significantly greater than for the nociceptive sensation ( $\mathrm{p}=0.001$, Bonferroni test). 


\section{Supplemental Table 1: Clinical data for human DRG donors.}

DRG tissue was obtained from Tissue For Research (donor 1 and 2) and the NIH NeuroBioBank (donor 3 and 4). Basic clinical data for each donor are summarized.

\begin{tabular}{|l|l|l|l|l|}
\hline donor ID & age & gender & race & cause leading to death \\
\hline 1 & 42 & female & white & chronic inflammatory demyelinating polyneuritis \\
\hline 2 & 68 & male & white & chronic obstructive pulmonary disease \\
\hline 3 & 48 & male & black & congestive heart failure \\
\hline 4 & 41 & male & white & atherosclerosis \\
\hline
\end{tabular}


Supplemental Table 2. Statistical analysis of areas $\left(\mathrm{cm}^{2}\right)$ of alloknesis, hyperalgesia, hyperknesis, wheal and flare/ or local erythema evoked by injection of a BAM8-22, ALA, HIS, a combination of BAM8-22 and ALA, and a combination of BAM8-22 and ALA and HIS.

\begin{tabular}{|c|c|c|c|c|c|}
\hline & BAM8-22 & ALA & HIS (*) & BAM8-22 + ALA & BAM8-22 + ALA + HIS (**) \\
\hline Alloknesis & $\begin{array}{l}4.0 \pm 1.2 \\
* p=0.000043 \\
* * p=0.04701\end{array}$ & $\begin{array}{l}3.7 \pm 0.9 \\
{ }^{*} p=0.000024 \\
* * p=0.03076\end{array}$ & $17.4 \pm 3.8$ & $\begin{array}{l}3.7 \pm 1.4 \\
* p=0.00002769 \\
* * p=0.03387\end{array}$ & $12.0 \pm 2.9$ \\
\hline Hyperalgesia & $\begin{array}{l}8.7 \pm 2.1 \\
* p=0.00009063 \\
* * p=0.001371\end{array}$ & $\begin{array}{l}5.7 \pm 1.4 \\
* p=0.0000006871 \\
* * p=0.00001542\end{array}$ & $21.0 \pm 3.8$ & $\begin{array}{l}10.6 \pm 2.5 \\
* \mathrm{p}=0.001512 \\
* * \mathrm{p}=0.01673\end{array}$ & $19.1 \pm 3.5$ \\
\hline Hyperknesis & $\begin{array}{l}7.2 \pm 1.9 \\
* p=0.01417 \\
* * p=0.007371\end{array}$ & $\begin{array}{l}5.4 \pm 1.5 \\
* p=0.001226 \\
* * p=0.0005838\end{array}$ & $15.4 \pm 2.9$ & $10.0 \pm 2.6$ & $15.9 \pm 3.2$ \\
\hline Wheal & $\begin{array}{l}0.5 \pm 0.1 \\
* \mathrm{p}<0.0000001 \\
* * \mathrm{p}<0.0000001\end{array}$ & $\begin{array}{l}0.3 \pm 0.0 \\
* p<0.0000001 \\
* * p<0.0000001\end{array}$ & $1.9 \pm 0.1$ & $\begin{array}{l}0.6 \pm 0.1 \\
* \mathrm{p}<0.0000001 \\
* * \mathrm{p}<0.0000001\end{array}$ & $1.9 \pm 0.2$ \\
\hline Flare/Erythema & $\begin{array}{l}5.4 \pm 1.5 \\
* p<0.0000001 \\
* * p<0.0000001\end{array}$ & $\begin{array}{l}1.2 \pm 0.7 \\
* p<0.0000001 \\
* * p<0.0000001\end{array}$ & $21.5 \pm 3.0$ & $\begin{array}{l}5.7 \pm 1.0 \\
* \mathrm{p}<0.0000001 \\
* * \mathrm{p}<0.0000001\end{array}$ & $21.0 \pm 2.6$ \\
\hline
\end{tabular}

*: Bonferroni post hoc p-values compared to HIS. **: Bonferroni post hoc p-values compared to BAM8-22 + ALA + HIS. Units are in $\mathrm{cm}^{2}$ and calculated as mean $\pm \mathrm{SEM}$. Data from all 29 subjects were included in the analysis. 